\title{
Diacronie
}

Studi di Storia Contemporanea

$\mathrm{N}^{\circ} 29,1$ | 2017

"Crash test"

\section{Teorie corporativiste nella Grecia del primo dopoguerra (1922-1940): dalla teoria alla prassi}

\section{Spyridon Ploumidis}

Traduttore: Stavros Kouroufexis

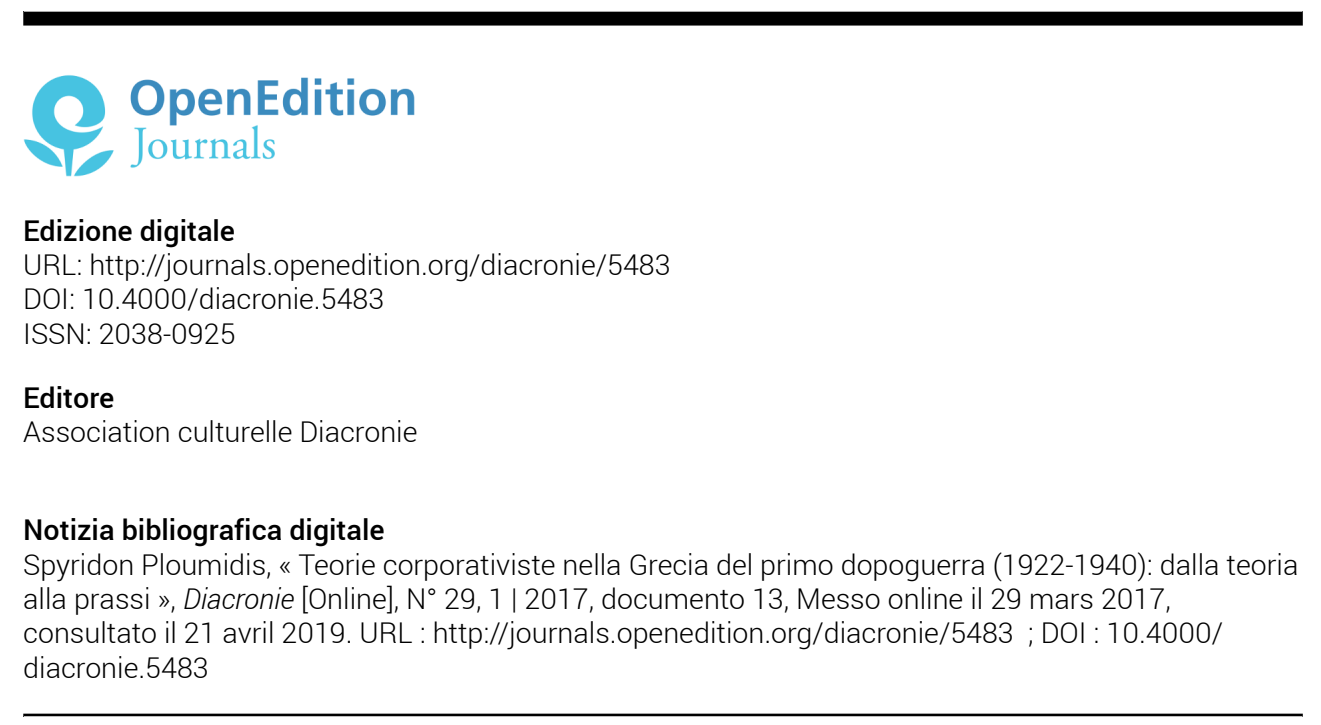

Creative Commons License 


\section{Diacronie}

Studi di Storia Contemporanea

29, 1/2017

"Crash test". Continuità, discontinuità, legami e rotture nelle dinamiche della storia contemporanea

\section{Teorie corporativiste nella Grecia del primo dopoguerra (1922- 1940): dalla teoria alla prassi}

\section{Spyridon PLOUMIDIS}

Per citare questo articolo:

PLOUMIDIS, Spyridon, «Teorie corporativiste nella Grecia del primo dopoguerra (1922-1940): dalla teoria alla prassi», Diacronie. Studi di Storia Contemporanea : "Crash test". Continuità, discontinuità, legami e rotture nelle dinamiche della storia contemporanea, 29, 1/2017, 29/3/2017,

URL: < http://www.studistorici.com/2017/03/29/ploumidis_numero_29/ >

Diacronie Studi di Storia Contemporanea $\rightarrow$ http://www.diacronie.it Rivista storica online. Uscita trimestrale.

redazione.diacronie@hotmail.it

Comitato di direzione: Naor Ben-Yehoyada - João Fábio Bertonha - Christopher Denis-Delacour - Maximiliano Fuentes Codera Anders Granås Kjøstvedt - John Paul Newman - Deborah Paci - Niccolò Pianciola - Spyridon Ploumidis - Wilko Graf Von Hardenberg

Comitato di redazione: Jacopo Bassi - Luca Bufarale - Gianluca Canè - Fausto Pietrancosta - Alessandro Salvador - Matteo Tomasoni Diritti: gli articoli di Diacronie. Studi di Storia Contemporanea sono pubblicati sotto licenza Creative Commons 3.0. Possono essere riprodotti e modificati a patto di indicare eventuali modifiche dei contenuti, di riconoscere la paternità dell'opera e di condividerla allo stesso modo. La citazione di estratti è comunque sempre autorizzata, nei limiti previsti dalla legge. 


\title{
13/ Teorie corporativiste nella Grecia del primo dopoguerra (1922-1940): dalla teoria alla prassi ${ }^{1}$
}

\author{
Spyridon PLOUMIDIS
}

Traduzione di Stavros KOUROUFEXIS

Nella Grecia del primo dopoguerra, analogamente a quanto accade in Italia o in Germania, si fa avanti un nuovo modo di concepire le relazioni tra Stato, economia e società: è il corporativismo "autoritario". Anche nel paese ellenico il crollo del liberalismo politico si era accompagnato ad una profonda crisi delle certezze sulla solidità e sull'utilità delle libertà in economia e aveva avuto un riflesso diretto nell'affermazione delle dittature di Kondylis e di Metaxas, fautori di una trasformazione dell'economia greca in senso dirigista. Questo articolo intende analizzare come, attraverso le novazioni normative e i tentativi di intervento nel sistema produttivo ellenico i poteri pubblici hanno tentato di allinearsi alle forme di controllo dell'economia che maturavano e si affermavano nel resto d'Europa.

\section{Il corporativismo come teoria}

Il corporativismo non è una teoria e ideologia sociopolitica casuale, ma insieme al capitalismo liberale e al socialismo è stato uno dei maggiori “-ismi” della moderna società industriale nel tardo XIX secolo e durante il primo dopoguerra ${ }^{2}$. Le idee corporativiste furono sviluppate come reazione ai problemi di difficile risoluzione causati dal capitalismo industriale e dal crollo graduale del ancien regime nell'Europa continentale. Corporativismo significa, molto semplicemente, la collaborazione fra lavoro e capitale ed è stato elaborato da intellettuali al fine di attenuare i conflitti di classe fra i capitalisti industriali e la classe operaia. Per raggiungere questa collaborazione c'era bisogno di un

\footnotetext{
${ }^{1}$ L'articolo è la traduzione dal greco dell'articolo successivamente comparso in lingua inglese sulla rivista «European History Quarterly». PLOUMIDIS, Spyridon, «Corporatist Ideas in Inter-war Greece: From Theory to Practice (1922-1940)», in European History Quarterly, 44, 1/2014, pp. 55-79.

${ }^{2}$ WILLIAMSON, Peter J., Varieties of Corporatism: A Conceptual Discussion, New York, Cambridge University Press, 1985, p. 4.
} 
elementare rapporto di solidarietà fra le due parti avverse. Le origini della teoria corporativista sono l'etica del cattolicesimo e il collettivismo del nazionalismo. La solidarietà predicata dalla Chiesa cattolica derivava dall'amore cristiano, mentre i teorici nazionalisti del corporativismo predicavano il sacrificio individuale a favore del bene collettivo nazionale - il corporativismo nazionale assunse dimensioni maggiormente autoritarie, in quanto del bene nazionale si faceva, in pratica, interprete un capo autoritario. In più gli ideatori del corporativismo idealizzarono l'immagine della società medievale e professarono la rinascita di questa idilliaca e armoniosa società dove regnavano valori etici superiori e solidarietà, la comprensione e l'assistenza reciproca ${ }^{3}$ : il corporativismo è una teoria idealista per eccellenza.

Oltre a quanto detto sopra il corporativismo presuppone la presenza di uno stato forte che soprintenda o che si assuma esso stesso l'incarico di organizzare i lavoratori in organizzazioni corporativiste ("sindacati"). I "sindacati" corporativisti, che si sostituiscono alle associazioni liberali di professione (unioni, circoli) dei lavoratori, devono possedere il riconoscimento e l'approvazione dello Stato e operano come istituzioni economiche e sociali intermedie, poste fra i lavoratori e lo Stato $^{4}$. I sindacati segnano la gestione orizzontale della rappresentanza professionale. Ogni sindacato include tutte le organizzazioni della medesima tipologia e professione, di lavoratori e impiegati, ma anche dei loro datori di lavoro. Ogni "sindacato" rappresenta un intero ramo (e uno soltanto), senza eccezioni, dell'industria, delle arti e delle professioni. Le organizzazioni sindacali professionali perdono il loro carattere verticale, e cioè classista, e ne assumono uno orizzontale e collettivista. Quindi il corporativismo sostituisce l'ordine economico-sociale individualista-liberale con uno collettivista e rigorosamente gerarchico. Il ruolo di garante del mantenimento di questa gerarchia viene assunto dallo Stato (rafforzato) ${ }^{5}$.

Peter Williamson ${ }^{6}$ ha individuato tre tipi di corporativismo: a) il corporativismo "consensuale" (consensual licenced corporatism); b) il corporativismo "autoritario" (authoritarian licenced); c) il "neocorporativismo" (neo-corporatism) ${ }^{7}$. In questo articolo verranno esaminate le prime due forme del corporativismo, le quali furono applicate durante il primo dopoguerra, mentre il cosiddetto "neocorporativismo" riguarda le economie industriali evolute dell'Occidente del secondo dopoguerra. Le differenze fra le prime due forme del corporativismo riguardano il tipo di controllo esercitato dai "sindacati" sui propri membri ed il rapporto fra i "sindacati" e lo Stato. Anche se in entrambi i sistemi corporativi lo Stato svolge, per definizione, una decisiva funzione di guida e di supervisione, nel primo caso le strutture della rappresentanza professionale e dell'organizzazione della produzione, permettono, in misura significativa, il consenso dei lavoratori per quel che riguarda i

\footnotetext{
${ }^{3}$ Ibidem, pp. 19-20, 22.

${ }^{4}$ Cfr. ROBERTSON, David, The Penguin Dictionary of Politics, London, Penguin Books, 1993, p. 117.

${ }^{5}$ WILLIAMSON, Peter, op. cit. pp. 20-21.

${ }^{6}$ Ibidem.

${ }^{7}$ Ibidem, pp. 7, 11.
} 
valori e gli obbiettivi del sistema ed un importante grado di autonomia della società nei confronti dello Stato ${ }^{8}$. Questa natura del corporativismo "consensuale" spiega la sua adozione da parte di regimi socialdemocratici come la Repubblica di Weimar (1919-33) ${ }^{9}$. Al contrario, il corporativismo “autoritario" è stato proprio dei regimi fascisti e "fascistoidi". L'attrattiva e l'ampia accettazione del corporativismo fra i regimi fascisti non sono dovute soltanto alla loro natura autoritaria, che in pratica si traduceva nel disciplinamento della classe lavoratrice e nella delegittimazione di ogni azione di sciopero, ma anche nel carattere sovra-nazionalista della loro ideologia. Inoltre, il fascismo e il corporativismo "autoritario" avevano gli stessi nemici: il liberalismo individualista, il socialismo materialista e, naturalmente, la lotta di classe. L'acuirsi dei conflitti sociali dopo la Prima guerra mondiale fece dell'adozione del corporativismo da parte dei fascisti italiani di Mussolini (il 1927 con la Carta del Lavoro) una necessità reale e non una questione metafisica ${ }^{10}$. Tuttavia, anche se il corporativismo viene considerato una componente del fascismo, questi due fenomeni politici non erano identici, ma avevano origini distinte ed ebbero un percorso diverso dopo la Seconda guerra mondiale.

\section{Conflitti di classe nella Grecia del primo dopoguerra}

Le condizioni economico-sociali nella Grecia del primo dopoguerra (1922-1940) favorivano la diffusione e l'applicazione delle idee corporativiste. Già dal primo decennio postbellico la Grecia conobbe una rapida crescita industriale (mediamente 6.8\% per gli anni 1922-1929) ${ }^{11}$. Questo aveva come risultato l'aumento della forza lavoro dell'industria da 154.633 (uomini e donne) nel 1917 a 278.855 nel $1930^{12}$. Le condizioni economico-sociali peggiorarono dopo la bancarotta ufficiale della Grecia, dichiarata da Eleftherios Venizelos il 27 aprile $1932^{13}$. L'industria greca, tuttavia, riuscì a riprendersi abbastanza presto e a raggiungere alti ritmi di crescita (in media 8\%) dal 1933 in poi (e fino alla fine del decennio) ${ }^{14}$. Negli anni 1931-39 vennero fondate 927 nuove imprese industriali ${ }^{15}$.

\footnotetext{
${ }^{8}$ Ibidem, pp. 9-11.

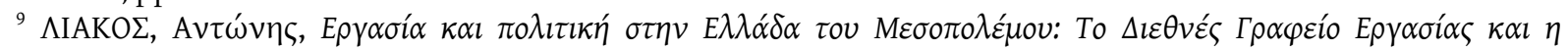

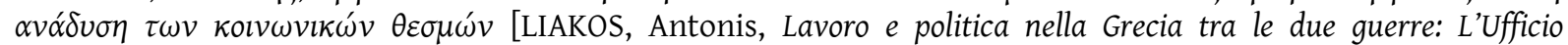

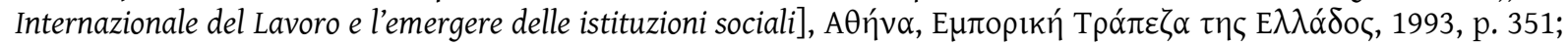
EYCK, Erich, A History of the Weimar Republic, vol. 2, From the Locarno Conference to Hitler's Seizure of Power, New York, J. Wiley, 1967, pp. 110-114.

${ }^{10}$ WILLIAMSON, Peter, op. cit., p. 77.

${ }^{11}$ MAZOWER, Mark, Greece and the Inter-War Economic Crisis, Oxford, Clarendon Press, 1991, pp. 91-92.

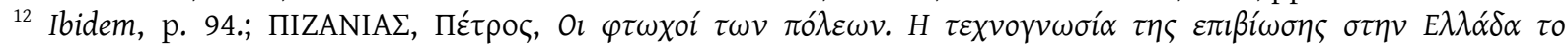

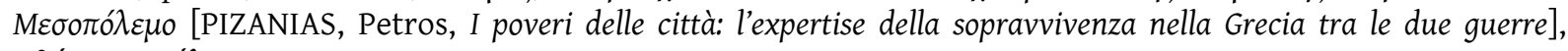

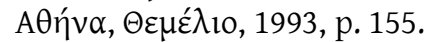

${ }^{13}$ MAZOWER, Mark, op. cit., pp. 172-173.

${ }^{14}$ Ibidem, pp. 237, 250-251

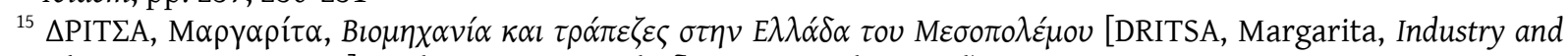

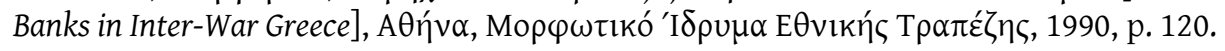


Tuttavia l'occupazione nel settore non crebbe altrettanto ma soltanto di 17.700 posti (da 157.300 nel 1930 a circa 175.000 dipendenti nel 1940, un aumento cioè di appena il $10 \%{ }^{16}$ ). In altre parole l'industria greca non poteva assorbire i nuovi ingressi nel mercato del lavoro e di conseguenza la disoccupazione endemica durante il secondo decennio postbellico rimase ferma al 9\%. Inoltre secondo la GSEE ( $\Gamma \Sigma E E$, Confederazione Generale dei Lavoratori Greci) la disoccupazione esplose con l'avvento della crisi economica mondiale e il numero dei disoccupati aumentò da 82.000 nel 1928 a 237.356 nel 1932, una cifra che corrispondeva al 35\% del totale della forza lavoro. Ciò era dovuto alla natura dell'industria greca, relativamente arcaica dal punto di vista della competitività, dei capitali, degli investimenti in nuove tecnologie, del personale specializzato, delle fonti di materie prime, dell'energia ecc. Nel 1936 il contributo del settore industriale al reddito nazionale era appena del $12 \%$.

Tutto ciò ebbe come risultato l'acuirsi dei conflitti di classe durante gli anni Trenta. La combattività della classe operaia sindacalizzata fu rafforzata dalla concentrazione geografica dell'industria greca. Nel 1930 il 59,6\% degli stabilimenti industriali e il 75,7\% della forza lavoro erano concentrati nella capitale, mentre le rispettive percentuali per la circoscrizione di Salonicco erano $26 \%$ e $18 \%{ }^{17}$. L'inasprimento dei rapporti di classe si deve anche ad un altro, soggettivo, fattore: nel novembre del 1931 venne imposta una nuova leadership nel Partito Comunista Greco (KKE) dall'Internazionale Comunista (Comintern); questa nuova leadership che faceva capo a Nicolas Zachariadis, seguendo le direttive staliniste, aveva indurito il suo atteggiamento nei confronti dell'ordine costituito borghese e dei suoi avversari socialisti ${ }^{18}$. Nello stesso anno (1931), i Circoli Operai di Salonicco, Drama e Kavalla (queste ultime due città erano i più grandi centri produttori di tabacco del paese), come anche quelli di Volos e di Larissa, caddero sotto il controllo della Unionista GSEE (fondata nel 1929), la quale veniva guidata dal $\mathrm{KKE}^{19}$. Nell'aprile-maggio del 1936 l'ondata di scioperi assunse il carattere di una marea. Il 9 maggio uno sciopero degli operai del tabacco di Salonicco portò a sanguinosi conflitti con la guardia rurale e l'esercito che finirono con l'uccisione di 9 manifestanti e il ferimento di oltre $200^{20}$. Il tumulto operaio-sindacalista non si fermò lì. Il 27 luglio

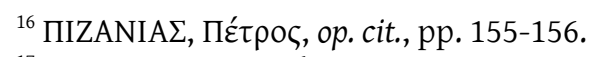

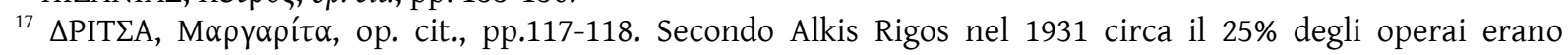

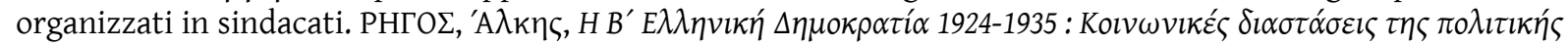

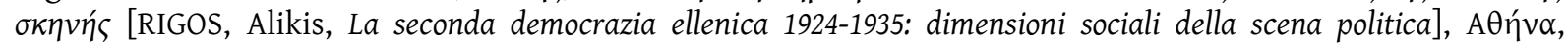
$\Theta \varepsilon \mu \varepsilon ́ \lambda ı, 1992$ p. 144

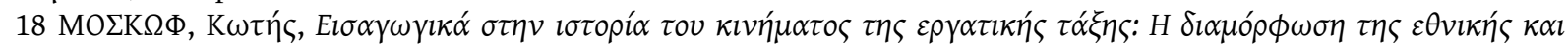

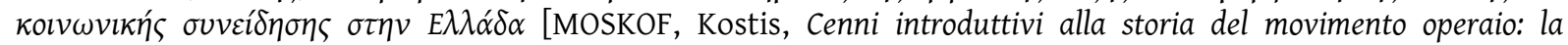

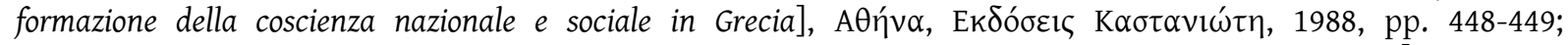

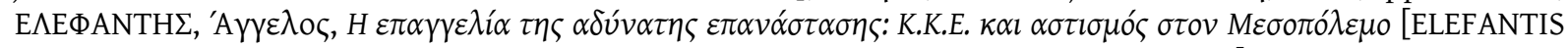

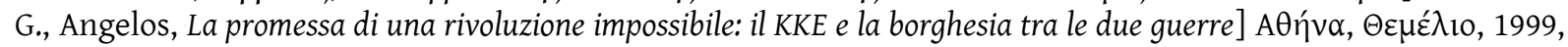
pp. 119, 357, 406-407.

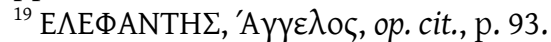

${ }^{20}$ Ibidem, p. 287; MAVROGORDATOS, George Th., Stillborn Republic: Social Coalitions and Party Strategies in Greece, 1922-1936, Berkeley-Los Angeles, University of California Press, 1983, pp. 147, 151-152; PAPACOSMA, Victor S.,
} 
i sindacati (GSEE e GSEE Unitaria ecc.) proclamarono uno sciopero generale di 24 ore per il 5 agosto in segno di protesta per l'intento del governo (allora ancora di tipo parlamentare e non dittatoriale, essendosi insediato il 13 aprile) di Ioannis Metaxas di promulgare l'arbitrato obbligatorio dello Stato nella conclusione dei Contratti Collettivi di Lavoro. La proclamazione dello sciopero generale diede a re Giorgio II e al primo ministro Metaxas il pretesto e la scusa per sospendere il funzionamento del Parlamento e di alcuni articoli fondamentali della Costituzione (del 1911) e di imporre un regime dittatoriale $^{21}$. Fra il 1936 e il 1940 il regime di Metaxas applicò (in misura limitata) alcune pratiche corporativiste, le quali copiavano il modello fascista e quello nazionalsocialista. Da questo punto di vista la Grecia tra le due guerre non è stata un'eccezione dalla norma europea. Prima di procedere ad un esame dettagliato della diffusione e dell'applicazione del corporativismo "autoritario", sarà utile guardare sinteticamente all'applicazione delle idee del corporativismo "consensuale" nel nostro paese.

\section{Il corporativismo "consensuale" in Grecia}

Le idee del corporativismo "consensuale" hanno avuto una diffusione limitata in Grecia. Prova di ciò è il fatto che le opere di Walter Rathenau ${ }^{22}$, di Emile Durkheim ${ }^{23}$ e di Mihail Manoilescu ${ }^{24}$ che furono le fonti fondamentali di queste teorie ${ }^{25}$, non sono mai tradotte (durante il primo dopoguerra) in greco. Questo vuoto (o silenzio) può essere spiegato, in parte, con il grande impatto che hanno avuto le idee del comunitarismo che è stato associato alla tradizione greca ${ }^{26}$. Il comunitarismo, come anche il corporativismo, offriva (in teoria) una via media fra il materialismo capitalista e comunista ed una soluzione idealista che avrebbe attenuato i contrasti di classe. Il comunitarismo era, secondo i suoi fautori locali (ad esempio Dinos Malouchos e Kostas Karavidas), compatibile con le tradizioni

Ioannis Metaxas and the "Fourth of August" dictatorship in Greece, in FISCHER, Bernd J. (ed.), Balkan Strongmen: Dictators and Authoritarian Rulers of South Eastern Europe, London, Hurst, 2007, pp. 165-198, p. 179.

${ }^{21}$ MAZOWER, Mark, op. cit., p. 289.

${ }^{22}$ RATHENAU, Walter, Der Neue Staat, Berlin, Fischer, 1919.

${ }^{23}$ DURKHEIM, Émile, De la division du travail social, Paris, Librairie Félix Alcan, 1893.

${ }^{24}$ MANOLEISCU, Mihail, Le siècle du corporatisme. Doctrine du corporatisme integral et pur, Paris, Editions Payot, 1934.

${ }^{25}$ WILLIAMSON, Peter, op. cit., pp. 26-27, 46-47, 58-59.

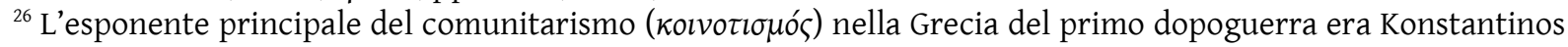
Karavidas (1890-1973), un ufficiale del Ministero degli Esteri. Il cuore della sua teoria era la 'comunità cooperativa autonoma' dei contadini, che «potrebbe ridare alla singola famiglia la vitalità che aveva perso a causa della sua dipendenza dallo stato iper-centralizzato ed il capitale». Il comunitarismo di Karavidas si

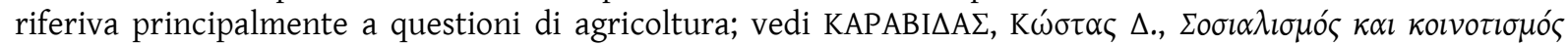

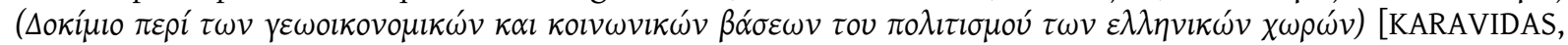
Kostas D., Socialismo e Comunitarismo (Studio sulle basi geoeconomiche e sociali della civilizzazione delle terre greche)],

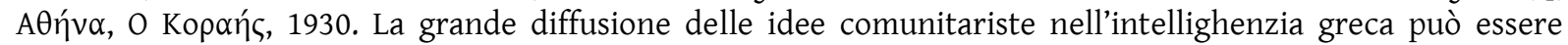
ricercata nella connessione del comunitarismo con l'antica filosofia aristotelica. TAM Henry, Communitarianism: A New Agenda for Politics and Citizenship, Houndmills, Basingstoke-London, Macmillan, 1998, pp. 18-19. 
dei sindacati e delle comunità agricole del periodo post-bizantino e di conseguenza avrebbero potuto offrire soluzioni più "genuine" e "praticabili" ai problemi dell'ellenismo contemporaneo ${ }^{27}$.

Il corporativismo "consensuale" venne applicato limitatamente all'istituzione senatoria, che era stato rifondata formalmente con la Costituzione Democratica del 1927 e aveva ripreso le sue funzioni nel 1929 (fino alla sua soppressione, operata dalla dittatura di Georgios Kondylis). Il dibattito sulla rifondazione del senato sotto la forma di un Consiglio Supremo dei professionisti e delle classi produttrici, come il Reichswirtschaftsrat della Repubblica di Weimar (il Reichswirtschaftsrat era stato fondato nel 1920 per la rappresentanza di "tutte le rilevanti professioni dipendenti e libere" come un mezzo per colmare il divario fra le classi sociali contrapposte) era iniziato già dal marzo del $1922^{28}$. Nel 1928 il professore di Diritto Costituzionale all'Università di Atene Alexandros Svolos, che era noto per le sue convinzioni socialdemocratiche, sostenne, in una sua pubblicazione indipendente, il principio della "rappresentanza professionale" (o, come da lui alternativamente chiamata, il principio della "rappresentanza degli interessi"), uno dei principi fondamentali del corporativismo. L'autore aveva, inoltre, dichiarato che questo principio seguiva la Wirtschaftsverfassung, il principio teorico su cui si era basata l'istituzione del Reichswirtschaftsrat. Il problema era l'«armonizzazione, sotto disciplina e ordine razionale, della vita economica, al fine di evitare la dominante anarchia nella produzione e nei rapporti economici», cosicché «gli interessi delle classi opposte» potessero «unirsi in uno sforzo comune». Nello stesso tempo Svolos precisò che rifiutava l'antidemocratico «Stato associazionista» e «l'organizzazione professionale» del «sistema assolutista fascista», e sostenne che «la rappresentanza degli interessi» in un «Senato Economico» dovrebbe basarsi sulla "partecipazione volontaria» e «sulla libertà di cooperazione», come era appropriato per una «democrazia borghese» ${ }^{29}$. L'anno seguente le idee di Svolos echeggiarono nella tesi sulla dittatura del giurista Michail Dendias, che sostenne ferventemente il principio della representation politique professionelle, autrement dite representation des interets, un principio che lui interpretò come una «rappresentanza sostanzialmente sindacale, basata

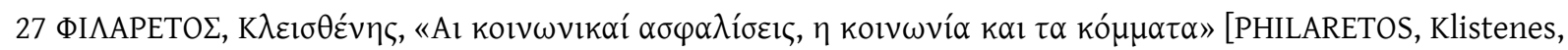

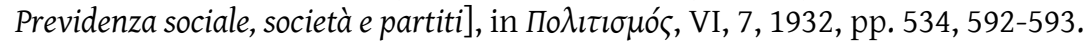

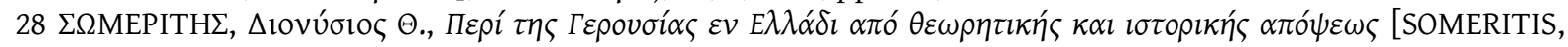

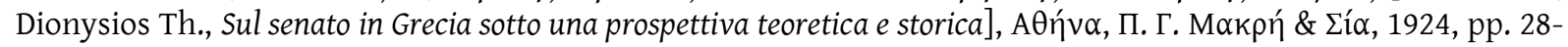
29, 131, 137-139; cfr. EYCK, Erich, op. cit., pp. 75-77.

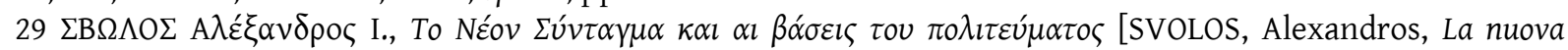

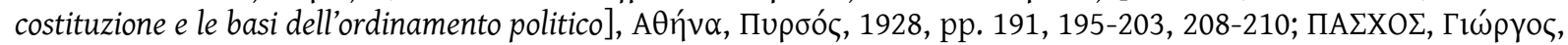

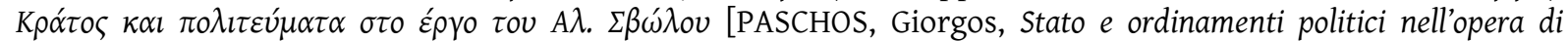

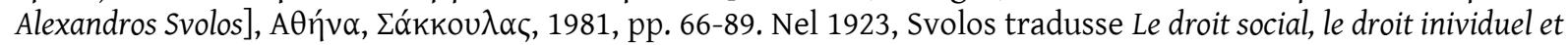
la transformation de l'État (Paris, F. Alcan, 1911), di Léon Duguit (professor di Diritto pubblico dell'Università di

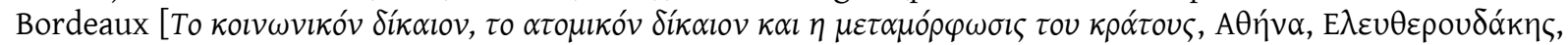
1923.]. Nella sua introduzione (pp. ii-iii), Svolos sosteneva che «la complessa organizzazione delle classi sociali» non può più essere basata su «lotta e violenza, come vorrebbero i marxisti ma sulla solidarietà sociale» e invocava «rappresentanza economicca delle classi sociali» all'interno di un «Parlamento delle professioni». 
sull'organizzazione sindacale» ${ }^{30}$. Alla fine il principio della «rappresentanza professionale» venne applicato in questo modo: $18 \mathrm{su} 120$ membri del Senato greco erano rappresentanti di organizzazioni professionali, come le Camere commerciali e professionali, le Camere Agricole, le Camere tecniche, L'Unione degli armatori Greci ecc ${ }^{31}$.

Riferimenti alle teorie corporativiste sono rinvenibili anche nell'opera di Georgios Theotokas (1905-1966), giurista ateniese e intellettuale liberale di rilievo negli anni Trenta che, successivamente (1944) aderì alla socialdemocrazia; nel gennaio 1932 Theotokas prevedeva, nel contest di un'economie dirigée, la trasformazione del Senato esistente in un'«assemblea economica» ${ }^{32}$.

\section{Il corporativismo "autoritario"}

\subsection{Nella teoria}

Le idee corporative "autoritarie", applicate da Ioannis Metaxas, avevano acquisito una basilare diffusione in Grecia poco prima del 1936. Alla fine del 1935 (cioè dopo l'instaurazione della dittatura di Kondylis) un ricercatore italiano aveva pubblicato una sua ricerca sullo «Stato sindacale», basata su una serie di lezioni da lui tenute all'Istituto Italiano di Cultura di Atene durante l'inverno di quell'anno. In questa sua ricerca aveva sviluppato le disposizioni principali della Carta del Lavoro (1927) e aveva spiegato i concetti di "corporazioni” e di Consiglio Nazionale delle Corporazioni. Il suo obiettivo dichiarato era quello che «il lettore possa farsi un idea precisa delle nuove idee politiche e sociali che provocarono la profonda riforma dello Stato Italiano e che oggi regolano la sua esistenza $»^{33}$. Nel 1937-1938 circolavano in greco altri tre studi sinottici sul sistema sindacale,

\footnotetext{
${ }^{30}$ DENDIAS, Michel, Le problème de la chambre haute et la représentation des intérêts à propos de l'organisation du Sénat grec, Paris, E. De Boccard, 1929, pp. 149-153, 303. La tesi di dottorato di Dendias è stata depositata all'Università di Parigi; dal 1930 Dendias intraprese una carriera brillante alle Università di Salonicco e di Atene occupando la cattedra di Diritto Amministrativo.

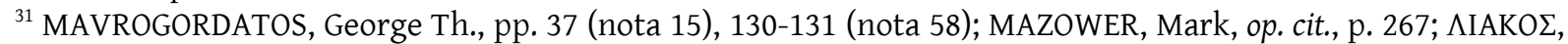

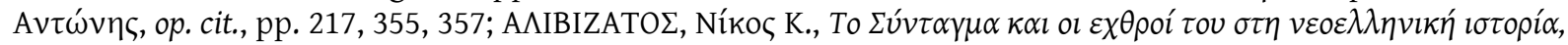
1800-2010 [ALIVIZATOS, Nikos K., La costituzione e i suoi nemici nella storia della Grecia contemporanea, 1800-2010],

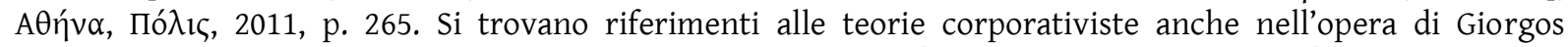

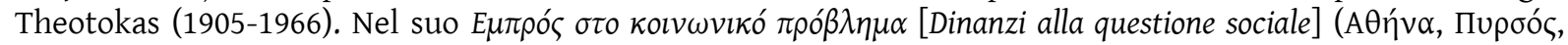
1932) l'intellettuale liberale radicale (che nel 1944 andò verso la socialdemocrazia), aspirò, nell'ambito di una economie dirigée (economia diretta), la trasformazione del Senato greco in «Parlamento Economico». Pensieri

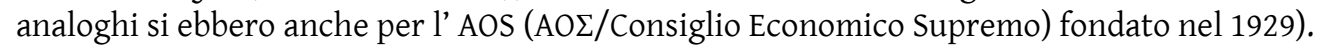

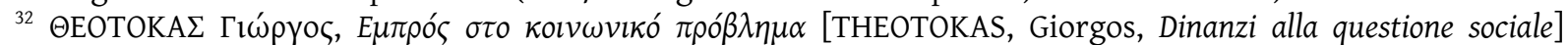

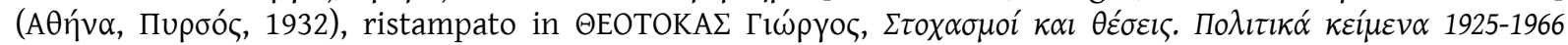

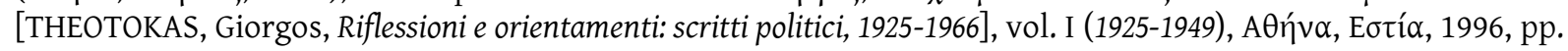

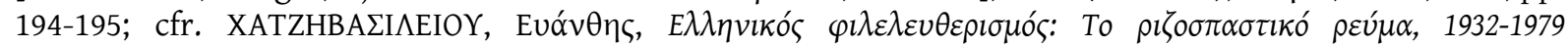

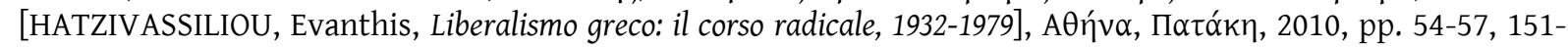
152.

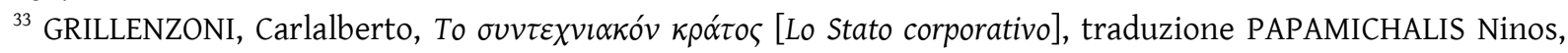

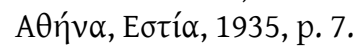


realizzati da Bruno Biagi (Sottosegretario alle corporazioni dell'Italia fino al 1935) e da Aristidis Vekiarelis (Ispettore della Banca Nazionale della Grecia ${ }^{34}$ ). Nel 1939 un avvocato ed ex deputato del partito dei Liberi pensatori di Ionnis Metaxas pubblicò un'opera voluminosa sull'economia diretta ed il sistema sindacale del fascismo e del nazionalsocialismo ${ }^{35}$.

I più ferventi fautori dello stato corporativo nel mondo accademico erano il professore di Economia Politica dell'Università di Salonicco Dimosthenis Stefanidis (1896-1975) e Ioannis Tournakis, professore di Economia Politica Applicata alla Scuola Suprema di Scienze Economiche e Commerciali. Circa un anno dopo l'instaurarsi del regime di Metaxas questi due economisti si dichiararono a favore dell'adozione del modello italiano ${ }^{36}$. Stefanidis, nello specifico, sostenne:

Davvero, se applicando il sistema economico liberale lasciamo alla propria sorte tutti i contrasti, acuti o meno, fra gli interessi economici, svaniranno gli interessi economici più deboli [...] la loro scomparsa ferirà nel profondo il corpo della società, diventando così una causa perenne di ostilità e di odio fra i vari fattori e classi produttrici. Al contrario, l'intervento dello stato, specie quando la sua organizzazione assicura l'avvento al potere dei "migliori” del popolo, di quelli che stanno al di sopra di classi e partiti, facilita il raggiungimento di vie di mezzo, e così l'indebolimento dei contrasti o anche la loro conversione in cooperazione degli interessi economici per il bene comune ${ }^{37}$.

Stefanidis (la cui opinione anti-liberale era evidente) sostenne che nel caso in cui il compromesso fra gli interessi economici non fosse possibile, l'intervento dello Stato avrebbe garantito che «il sacrificio degli interessi economici» sarebbe stato «di minore importanza per la collettività».

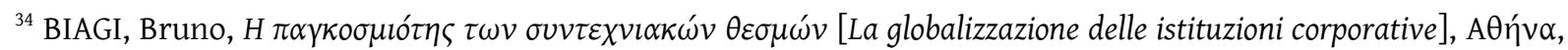

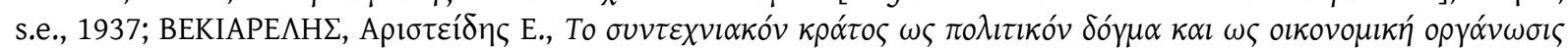
[VEKIARELIS, Aristidis, The Corporatist State as a Political Doctrine and as an Economic Organization], AӨńval,

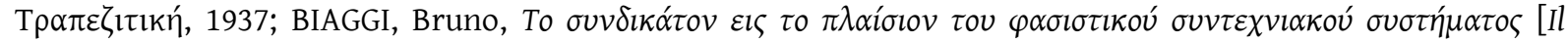

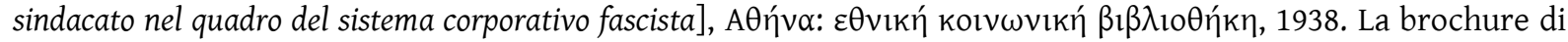
Biaggi si basava su una sua lezione tenuta all'Istituto Italiano di Atene. Il sistema corporativo italiano veniva

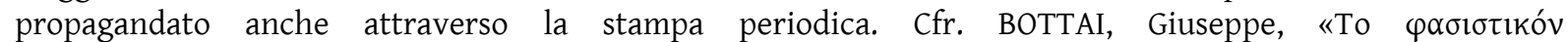

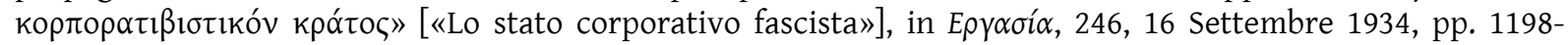

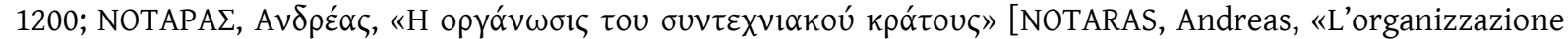

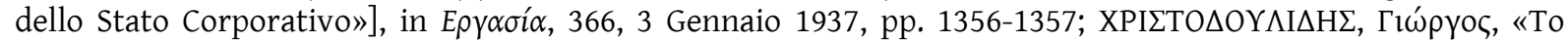

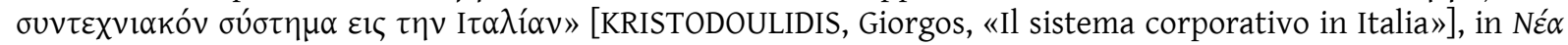

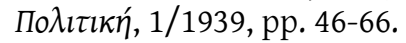

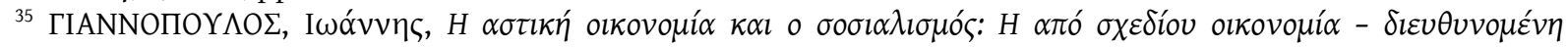

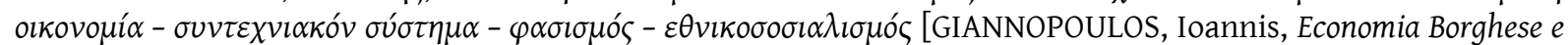
socialismo: l'economia pianificata - l'economia controllata - il sistema corporativo - fascismo - nazionalsocialismo], AӨท́vo, s.e., 1939.

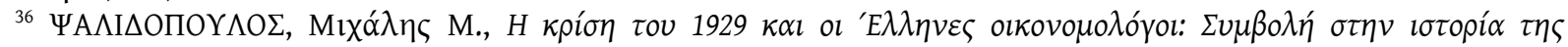

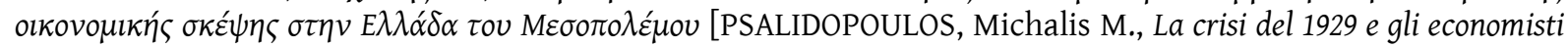

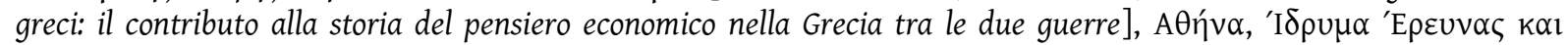

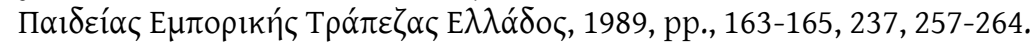

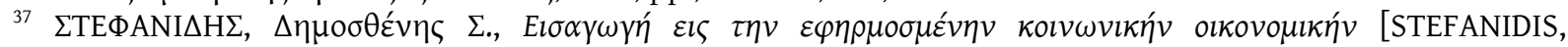

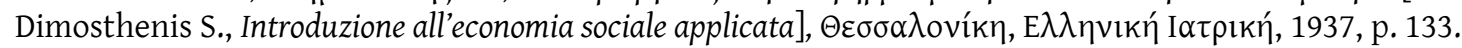


Stefanidis ribadì, successivamente, ancora una volta che il «considerevolmente più ampio intervento dello Stato nell'economia sociale, soprattutto al fine di rendere la massima giustizia economica e sociale sarebbe divenuto possibile, facilitando l'avvento al potere dei "migliori" del popolo, dal punto di vista dell'istruzione, della capacità amministrativa e soprattutto della moralità $»^{38}$. Su questo Stefanidis rinviò esplicitamente all'opera di Robert Michels Le partis politiques: essai sur les tendances oligarchiques des democraties ${ }^{39}$ il quale si dichiarava favorevole ad un'aristocrazia politica elitaria ${ }^{40}$. L'inquadramento politico del corporativismo proposta da Stefanidis era largamente anti-liberale, anti-parlamentare e autoritaria: «La cosiddetta democrazia parlamentare non sembra più capace di rispondere alla necessità dell'intervento statale attivo nell'economia sociale, come una forma di governo di altri tempi, altre condizioni sociali ed economiche [...] i popoli avanzati e tesi al progresso devono adattare la propria organizzazione statale alla suddetta necessità (di intervento attivo dello Stato) e affidare per questa ragione - che non è la sola - il potere a gruppi "aristocratici" stanti al di sopra di classi e partiti»"

Stefanidis definì il corporativismo (cioè questo tipo di intervento statale nell'«economia sociale») come la «aurea via di mezzo fra individualismo e comunitarismo» (cioè fra l'economia del liberalismo classico e quella del socialismo), e lo classificò nel quadro di un'«economia popolare (cioè völkisch) $»^{42}$. Il professore greco trasse le sue idee per la costituzione dello Stato Corporativo da Erwin von Beckerath ${ }^{43}$, il quale - fra l'altro - aveva sostenuto che «lo stato liberale appartiene al passato ${ }^{44}$. Infine, Stefanidis propose l'adozione da parte dei greci della politica economica dell'Italia fascista e della Germania nazionalsocialista. In particolare, in questa sua pubblicazione, mise in evidenza lo Stato Corporativo di Ugo Spirito, la Carta del Lavoro del 1927 e i concetti di sindacati, corporazioni, unioni, confederazioni ecc. mentre rinviò anche al Mein Kampf $f^{45}$. L'economista greco si muoveva, ovviamente, nella direzione del corporativismo «nazionale/autoritario». Dichiarò che «l'obbiettivo più alto dell'organizzazione sindacale è la nazione»: questa «politica economica e sociale sarebbe più correttamente caratterizzata come nazionale, perché nacque principalmente dalla contemporanea esaltazione del sentimento nazionale in un numero rilevante di paesi, e fu con esso congiunta. [...] Questa politica economica, contrariamente allindividualismo e al cosmopolitismo della scuola liberale, sventola la bandiera dell'interesse economico e ha come fine

\footnotetext{
${ }^{38}$ Ibidem, pp. 133-134, 138.

${ }^{39}$ MICHELS, Robert, Le partis politiques: essai sur les tendances oligarchiques des democraties, Paris, Flammarion, 1914.

${ }^{40} \Sigma$ TEФANI $\Delta \mathrm{H} \Sigma, \Delta \eta \mu \circ \sigma \theta \varepsilon ́ v \eta \varsigma \Sigma$., op. cit., pp. 45-55.

${ }^{41}$ Ibidem, p. 140.

${ }^{42}$ Ibidem, p.138.

${ }^{43}$ Von BECKERATH, Erwin, Die Idee der korporativen Wirtschaft, in DOBBERT, Gerhard [hrsg. von], Die fasistische Wirtschaft: Probleme und Tatsachen, Berlin, Hobbing, 1934

${ }^{44} \Sigma$ TEФANI $\Delta \mathrm{H} \Sigma, \Delta \eta \mu 0 \sigma \theta \dot{v} \eta \varsigma$ $\Sigma$., op. cit., pp. 138, 140-141.

${ }^{45}$ Ibidem, pp. 86-89, 97.
} 
l'infusione di idee nazionaliste» ${ }^{46}$. Da parte sua, Ioannis Tournakis propagandava sistematicamente

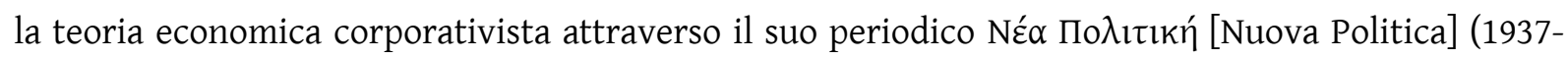
1940) suggerendo la rottura totale con la tradizione economica liberale e la svolta radicale verso la politica dell'autarchia, l'adozione totale del sistema corporativo italiano ${ }^{47}$.

La pubblicazione volgarizzata più completa della teoria corporativista "autoritaria" per il pubblico greco è stata fatta da Georgios Merkouris, ex ministro dell'Economia Nazionale e capo del minuscolo Partito Nazional Socialista Greco (fondato nel 1933). Già nel 1933 Merkouris aveva suggerito la regolamentazione dei rapporti di classe «in maniera realmente giusta sotto la sorveglianza insonne delle Leggi» cosicché cessasse la «concorrenza ostile fra le classi»e dominassero i principi dell'«Unità Nazionale», della «Serenità» e della «Disciplina Sociale» ${ }^{48}$. Nel 1936 nella sua breve pubblicazione Lo Stato Corporativo, Merkouris aspirava, "grazie alla sua grande attualità, alla più ampia diffusione e divulgazione del dogmatismo scientifico, da cui derivò la formazione di un moderno sistema amministrativo, quello del Corporativismo e, da esso, dello stato Corporativo basato sulla realtà della finanza pubblica e dell'economia» ${ }^{49}$. L' autore definì lo Stato Corporativo come il contrario «dell'individualismo, del capitalismo e del comunismo» e come la «più perfetta espressione della ragionevole difesa sociale derivante da imperative necessità economiche e politiche, le quali sono il risultato degli abusi del liberalismo e delle condizioni postbelliche» ${ }^{50}$. Merkouris definì il corporativismo come una creazione del fascismo italiano e come una parte inseparabile del «nuovo ordine» che era uscito «dalle mura di Roma» e che si espandeva «nel mondo» (in Portogallo, in Austria, in Germania ecc.), assumendo «oggigiorno una forma mondiale» ${ }^{51}$. L'autore si rivolse anche contro le «utopie comuniste» e «la lotta di classe» come un'«insopportabile situazione scissionista, la quale ha favorito il socialismo violento e il comunismo», mentre dall'altra parte parlò dei «fallimenti clamorosi del Parlamentarismo». Il colmarsi del divario che veniva causato «dal conflitto fra gli interessi del Capitale e del Lavoro» sarebbe stato operato dall' «insolubile anello di coesione, la Nazione» ${ }^{52}$.

\footnotetext{
${ }^{46}$ Ibidem, pp. 81-82, 91.

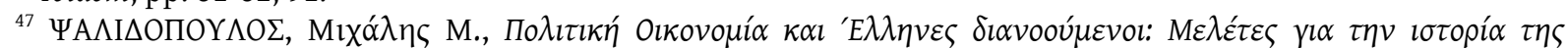

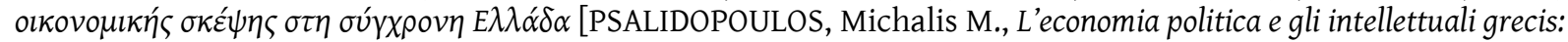

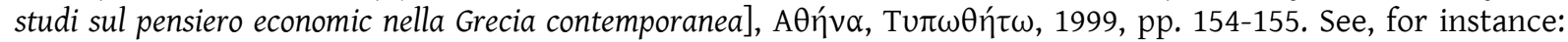

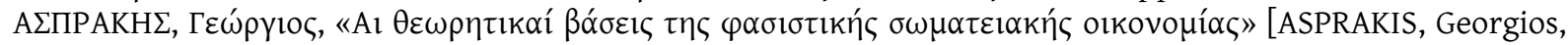
«Le basi teoretiche dell'economia corporativa italiana»], in Nea Politiki, 1/1937), pp. 97-105; XPI $\Sigma T O \Delta O Y \Lambda I \Delta H \Sigma$, Гı́́pүos, op. cit.

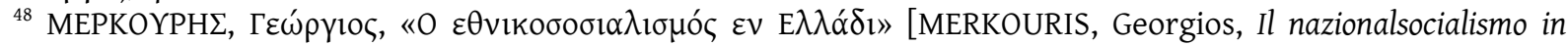

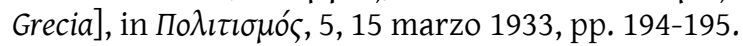

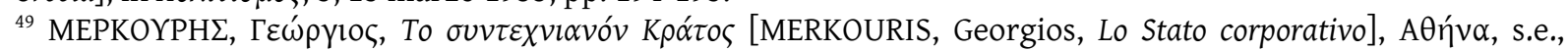
1936, p. 5.

${ }^{50}$ Ibidem, p. 6.

${ }^{51}$ Ibidem, pp. 7, 11, 14, 16.

${ }^{52}$ Ibidem, pp. 8-9.
} 
Merkouris aveva spiegato in parole semplici che «Stato Corporativo» è «la traduzione del termine Corporativismo», mentre «il termine corporation potrebbe e dovrebbe essere tradotto con la corrispondente parola greca sindacato». Il termine «sindacato», spiegò, è «lincorporazione in una sola unione di rappresentanti di sindacati agricoli, operai meccanici, industriali, commercianti e di qualsiasi altro settore, tutti quanti cooperanti nello stesso ramo produttivo o industriale». A questo punto Merkouris precisò che il «sindacato costituisce quell'unione consistente in rappresentanti dei sindacati riconosciuti», cioè dei sindacati istituzionalmente riconosciuti dalle autorità sindacali dello Stato e non qualsiasi organizzazione sindacale e professionale libera. In più, seguendo il paradigma del «sistema corporativista italiano», spiegò il significato di «categoria»: «La categoria include la somma di tutti quelli che esercitano la medesima funzione professionale per la produzione di un determinato bene, esercitano la stessa professione, indipendentemente dal fatto che siano o meno iscritti al sindacato». Tuttavia «la legge (italiana dello Stato fascista di Mussolini) dice che il sindacato riconosciuto rappresenta l'intera categoria». Di conseguenza il sindacato riconosciuto rappresenta l'intera categoria di una data zona, in modo che possa concludere accordi collettivi, aventi vigore per la categoria intera». Così diventa comprensibile l'indicazione dell'autore, per cui «le corporation (cioè i sindacati) sono organi dello Stato». In altre parole anche se l'iscrizione dei lavoratori ai sindacati riconosciuti dallo Stato non era obbligatoria, almeno in teoria, era tuttavia obbligatoria nella prassi. Dal momento che i sindacati (cioè le organizzazioni sindacali di primo grado) divenivano, nell'ambito del corporativismo, personalità giuridiche di diritto pubblico, la loro funzione seguiva l'articolazione amministrativa dello Stato: per ogni zona poteva esserci «un sindacato riconosciuto». In seguito Merkouris trattò brevemente del terzo grado dell'organizzazione sindacale, del cosiddetto Consiglio Nazionale dei Sindacati o Consiglio Sindacale, sul quale sovrintendeva il Ministero dei Sindacati e del Lavoro. Infine, l'ex Ministro chiarì ai suoi lettori che «la (libera) organizzazione sindacale è verticale, mentre quella corporativista è orizzontale». La sua proposizione politica era questa: «scioltisi tutti i Sindacati, Organizzazioni e Circoli, dovranno reistituirsi sotto la denominazione "Sindacati", nel senso e la sostanza giuridica che ad essi darà l'applicazione del corporativismo [N.d.A. in Grecia]». Merkouris concluse che «non può, l'interesse personale, essere messo al di sopra dell'interesse della Nazione e dell'interesse generale della produzione». Di questo tipo erano le norme «socialmente etiche» che il sistema corporativista aspirava ad instaurare, una «funzione armonizzante» nell'economia o anzi un «sistema di un'economia disciplinata e quindi controllata». Per lui il lavoro era un «dovere sociale» e quindi doveva obbedire a norme regolatrici. In questo sistema il ruolo di regolatore supremo spetterebbe allo Stato sotto la forma dello «Stato Etico» di Mussolini. Ma l'interventismo dello Stato nell'economia e nei rapporti lavorativi non sarebbe stato esteso alla produzione in sé (cioè lo Stato non avrebbe nazionalizzato i mezzi di produzione trasformandosi in datore di lavoro), ma si sarebbe limito ad un ruolo regolatore e organizzativo: «Attraverso l'economia corporativista arriviamo, 
senza dubbio, ad un maggiore interventismo dello Stato nell'economia, ma questo consiste in un suo intervento non in quanto produttore, bensì in quanto organizzatore» ${ }^{53}$.

\subsection{Il corporativismo "autoritario" nella pratica}

Copie delle pubblicazioni di Grillenzoni, di Biagi, di Vekiarelis, di Stefanidis e di Giannopoulos si possono trovare nella biblioteca personale di Ioannis Metaxas, che è conservata nella Biblioteca del Parlamento Greco $^{54}$. Tuttavia nel programma politico iniziale del partito dei Liberi pensatori (fondato nell'ottobre 1922) non c'era nessun riferimento al corporativismo; al contrario veniva rifiutata «totalmente qualsiasi riduzione delle libertà popolari» e si sosteneva la «totale libertà personale e politica» e «ogni sorta» di (libere) cooperative agricole ${ }^{55}$. Il primo pubblico annuncio politico di misure corporativiste ("autoritarie") fu fatto circa un anno dopo la bancarotta del paese nel 1932 (ed il conseguente inasprimento dei rapporti di classe), ed esattamente un mese dopo la disfatta schiacciante del venizelismo a seguito del fallito golpe di Plastiras del 3 marzo 1933 e più specificamente nelle dichiarazioni programmatiche del secondo governo di Panagis Tsaldaris nell'aprile del 1933 al Parlamento. In altre parole l'ingresso del corporativismo nella prassi politica greca andò di pari passo con il declino del liberalismo economico e (soprattutto) politico ${ }^{56}$. Tsaldaris annunciò che avrebbe perseguito l'innalzamento etico e materiale dell'operaio e la sua armoniosa collaborazione con il capitale imprenditoriale, che avrebbe anche protetto e che, «al fine di attenuare ogni probabile contrasto acuto fra di loro, dinanzi allo scoppio di qualsiasi sciopero da parte dei lavoratori o dei datori di lavoro, avrebbe suggerito che fossero applicate misure analoghe a quelle vigenti in altri stati ${ }^{57}$. Lo stesso Ioannis Metaxas, in una sua intervista al giornale «Kathimerini» (6 gennaio 1934) si dichiarò favorevole al "più intensivo interventismo dello Stato" nell'economia e nella società capitalista», mentre dichiarò allo stesso tempo che «il sistema parlamentare» era ormai «totalmente incapace di rispondere ai nuovi problemi, sorti nella vita dei popoli $\aleph^{58}$. Nelle dichiarazioni programmatiche $(25$ aprile 1936$)$ del suo primo governo (parlamentare) Metaxas rese noto il suo intento di ottenere «possibilmente più solidi termini di collaborazione fra capitale e lavoro»; considerò come una delle misure principali per il successo di

\footnotetext{
${ }^{53}$ Ibidem, pp. 12-26, 31-32.

${ }^{54}$ URL: < http://catalog.parliament.gr > [consultato il 28 gennaio 2017].

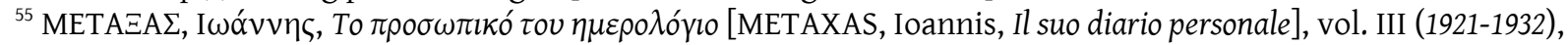

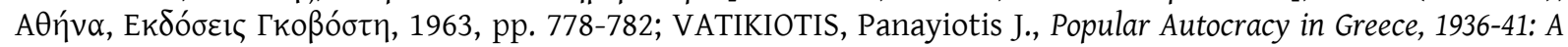
Political Biography of General Ioannis Metaxas, London-Portland (OR), 1998, pp. 121-122.

${ }^{56}$ Per le consequenze del colpo di Stato del 6 marzo 1933, si veda MAVROGORDATOS, George Th., op. cit., 338341.

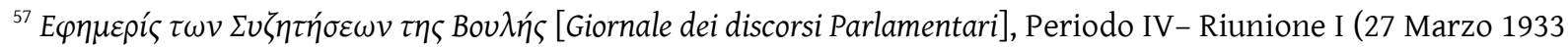
- 30 Gennaio 1934), Atene 1934, p. 9.

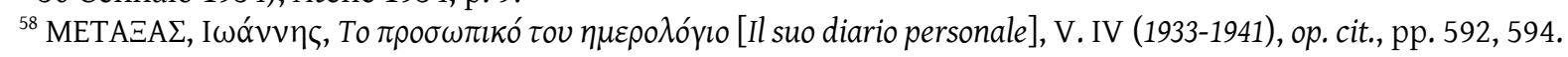


questo obiettivo «l'ampia applicazione dei contratti collettivi di lavoro» e la «regolamentazione organizzativa da parte dello stato delle questioni derivanti dai collettivi conflitti di lavoro» ${ }^{59}$.

Allo stesso modo le idee corporative non erano incluse neanche nel programma politico iniziale del Partito Radicale Nazionale del generale Georgios Kondylis (fondato nell'agosto del 1932) ${ }^{60}$. Tuttavia dopo il golpe e la presa di potere (10 ottobre 1935), Kondylis (un sostenitore dichiarato di Mussolini, che incontrò due volte nel luglio del $1935^{61}$ ) dichiarò, in un suo discorso pubblico, il 21 ottobre, «la realizzazione della collaborazione fra capitale e lavoro» come uno dei pilastri fondamentali della sua politica sociale ${ }^{62}$.

Il primo passo verso l'applicazione di misure corporative in Grecia fu fatto con l'istituzione (per la prima volta nella storia del paese) del Ministero del Lavoro, il 14 ottobre $1935^{63}$; questo ministero venne istituito come uno strumento di esercizio di una politica "sindacale". Alla fine di agosto, Metaxas declassò il Ministero del Lavoro in Dipartimento e lo collocò sotto la sovrintendenza del Ministero dell'Economia nazionale. Nello stesso tempo, però, aggiunse alla sua legge costituente la precisazione che le competenze del suddetto ministero includevano la tutela «in favore dell'assicurazione di una collaborazione armoniosa fra lavoro e capitale al fine di promuovere l'Economia Nazionale e per il sollevamento morale e materiale delle classi lavoratrici», come anche «l'intervento arbitrario per la risoluzione delle dispute fra lavoratori e datori di lavoro, derivanti da contratti, costumi o consuetudini lavorative» ${ }^{64}$. Il secondo strumento più importante per l'applicazione della politica corporativa "autoritaria" era la promulgazione dei contratti di lavoro collettivi, i quali determinavano «il livello minimo dei salari degli impiegati privati e delle paghe giornaliere degli operai industriali». I contratti di lavoro collettivi che, inizialmente vennero promulgati con una legge obbligatoria del governo Kondylis, introdussero la «procedura dell'arbitrato obbligatorio» nella conclusione di questi contratti collettivi come anche in qualsiasi

\footnotetext{
${ }^{59}$ Ibidem, p. 206.

${ }^{60}$ In vista delle elezioni nazionali del settembre 1932 il Partito Nazionale Radicale aveva come obiettivo, nel campo della "politica sociale", «la protezione degli economicamente deboli nei confronti dagli economicamente potenti, l'abbattimento degli sfruttamenti e dei privilegi sociali ingiusti, in particolar modo attraverso lo sviluppo della legislazione operaia (assicurazioni sociali)», e si poneva a favore della «necessità sociale di protezione delle classi lavoratrici, le quali rischiano di cadere nella completa dipendenza dal capitale». In un suo discorso a Kallithea (17 Settembre 1932), Kondylis si impegnò a perseguire la «liberazione del popolo lavorante dallo sfruttamento del Capitale» e a proteggere il lavoro. Si veda: NÉov Kpátos, 5, 1

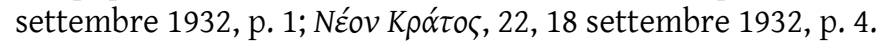

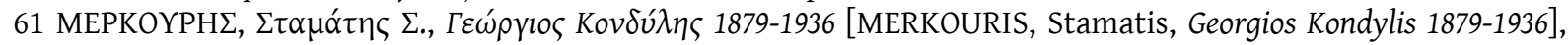

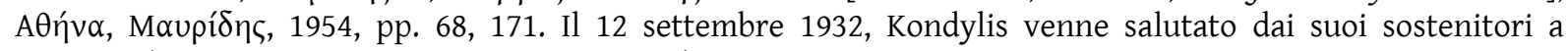
Katerini (una città nella Grecia settentrionale) come "il Mussolini di Grecia". Si veda: Neon Kratos, 17, 13 settembre 1932, p. 4.

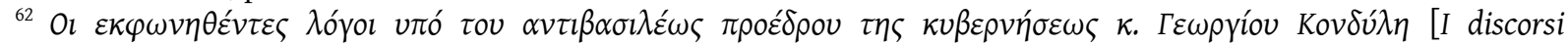

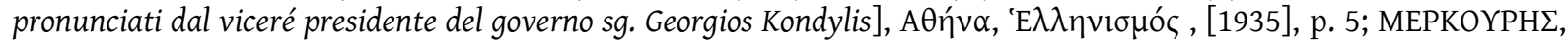

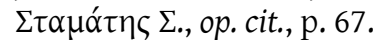

${ }^{63}$ Ephimeris tis Kyverniseos [Giornale del governo], Serie A, N. 465, 14 ottobre 1935, p. 2313; N. 536, 8 November 1935, p. 2614.

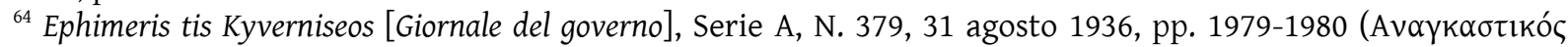

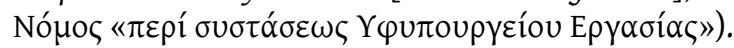


disputa fra datori di lavoro e salariati del settore privato, in aziende dove la forza lavoro superava le 10 persone $^{65}$. La procedura dell'arbitrato obbligatorio incontrò tuttavia le obiezioni e la reazione tanto dei lavoratori quanto dei datori di lavoro ${ }^{66}$. La proclamazione dello sciopero nazionale operaio per il 5 agosto 1936 dalla GSEE e la GSEE Unitaria aveva come rivendicazione principale la non applicazione del decreto legislativo per l'arbitrato obbligatorio dello Stato nelle dispute lavorative, che era stato votato dal consiglio dei ministri il 21 luglio ${ }^{67}$. Alla fine l'applicazione di questi contratti è stata compiuta durante la dittatura di Metaxas, quando vennero firmati (12 agosto) «da parte del governo e dei rappresentanti delle organizzazioni dei datori di lavoro e dei lavoratori» e vennero ratificati tramite un decreto emergenziale (7 settembre) i primi due "contratti di lavoro collettivi». La legge in questione precisò anche l'universalità della loro vigenza: «La sottoscrizione di questi contratti si intende come totalmente sostitutiva alla loro sottoscrizione da qualsiasi altra organizzazione lavorativa o dei datori di lavoro, generale, locale o speciale, e vincola anche queste organizzazioni insieme alle sottoscritte Organizzazioni Generali per la durata menzionata nei contratti $^{68}$. Secondo il propagandistico punto di vista ufficiale del regime, l'applicazione del sistema dei contratti collettivi "Nazionali" del lavoro "ha armonizzato gli interessi dei fattori che lo rappresentano [N.d.T. il lavoro] e ha restituito ad esso il suo valore vero come di un fondamentale ufficio sociale» ${ }^{69}$. Allo stesso tempo il Dipartimento della Stampa precisò il carattere vincolante di questi contratti per tutti i lavoratori del settore privato: «la definizione della paga giornaliera minima non è stata limitata soltanto ai grandi rami di certe industrie, ma assunse un'estensione quasi totale su tutto il territorio e in tutte le professioni» ${ }^{70}$. I primi due contratti collettivi regolavano i livelli minimi della paga giornaliera degli operai industriali e i limiti minimi dei salari mensili «per i dipendenti dei negozi commerciali, degli uffici, delle Aziende Anonime e dei dipendenti privati in genere». Alla loro promulgazione il «mondo dei datori di lavoro» fu rappresentato dai presidenti delle Camere commerciali e industriali di Atene, Pireo e Salonicco, dal presidente dell'Associazione degli Industriali Greci, da rappresentanti dell'Unione delle Banche Greche, dai Circoli Commerciali di Atene e del Pireo, come anche da un membro del Consiglio Economico Supremo. La classe operaia e quella impiegatizia furono rappresentate rispettivamente dal presidente del Centro Operaio di Atene e da un rappresentante del Centro Operaio di Pireo, dal

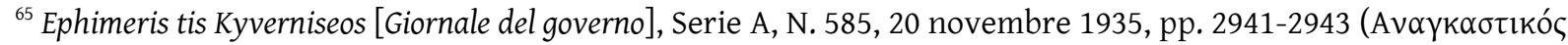

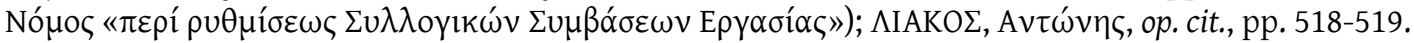

${ }^{66}$ MAZOWER, Mark, op. cit., p. 268.

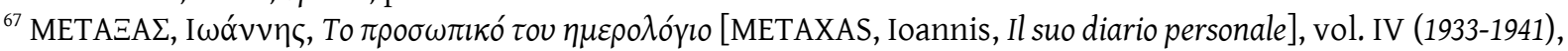
op. cit., pp. 219-220.

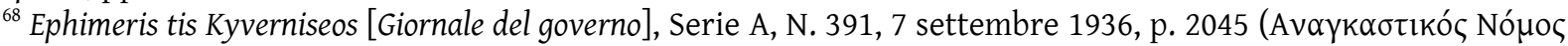

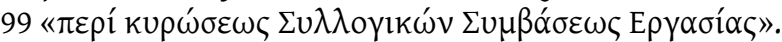

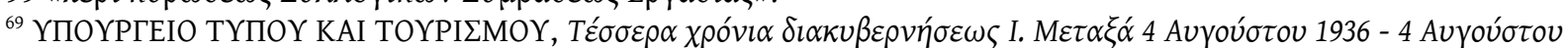
1940 [DIPARTIMENTO PER LA STAMPA E IL TURISMO, Quattro anni di governo di I. Metaxas 4 agosto 1936 - 4

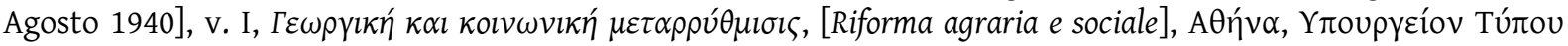

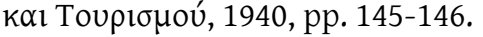

${ }^{70}$ Ibidem, p.147.
} 
presidente dell'Unione degli operai carico-scarico del porto di Pireo, dal presidente dell'Unione Panellenica Ingegneri delle fabbriche industriali, dal presidente dell'Unione dei tessitori di Atene, dal Presidente dell'Associazione degli Impiegati Commerciali di Atene e da un rappresentante degli insegnanti privati ${ }^{71}$. Con il decreto emergenziale 1435 dell'ottobre 1938, la GSEE (la quale all'epoca era naturalmente sotto il controllo del regime) venne autorizzata a «rappresentare tutte, in generale, le organizzazioni rappresentative operaie e impiegatizie agli organi collettivi dell'amministrazione e dell'assicurazione ai tribunali d'arbitrato e assicurativi, alla promulgazione di ogni sorta di contratto di lavoro collettivo in generale e ad aiutare le organizzazioni ad essa sottoposte per l'armonizzazione gli interessi dei fattori che costituiscono il lavoro nazionale» ${ }^{72}$. Nello stesso anno Stefanidis espresse la sua approvazione per «l'alquanto efficace sforzo di regolazione dei rapporti fra gli industriali e i loro dipendenti e della creazione di terreno per la vera collaborazione fra questi fattori per il bene della collettività»; in questo "sforzo" incluse in primo luogo la sottoscrizione dei contratti collettivi sotto l'egida dello Stato. «Grazie a questo sforzo», il professore di Economia politica vedeva «diretto verso la sua scomparsa totale il punto forse più oscuro del nostro progresso industriale, cioè la tensione fra datori di lavoro e lavoratori, l'infinita serie di scioperi e controscioperi e l'eterno turbamento della nave sociale che, fino a questi ultimi tempi navigava serena» ${ }^{73}$. È ridondante notare qui che l'imposizione del regime del 4 agosto venne accompagnato dal divieto di sciopero, di picchettaggio e di altre manifestazioni che impedivano il processo produttivo ${ }^{74}$.

Infatti il regime di Metaxas compì una rottura radicale con il sistema lavorativo liberale del passato. Nel suo discorso per l'inaugurazione dell'undicesima fiera di Salonicco (6 settembre 1936) Metaxas sottolineò che oramai «lo Stato prende in mano la direzione dell'economia dei paesi e il controllo dell'economia viene da esso esercitata appieno». In una sua intervista alla «Völkischer Beobachter» di Berlino del 24 settembre 1936, Metaxas, facendo un passo ulteriore dichiarò che «il nuovo Stato sarà sindacale», e che intendeva creare «organizzazioni di datori di lavoro e di lavoratori in ciascuna regione $»^{75}$, ma questa sua dichiarazione non si concretizzò mai. Nel novembre del 1936 il Ministro dell'Economia Konstantinos Zavitzianos annunciò la creazione di un Gran Consiglio del Lavoro Nazionale, ma senza dare ulteriori informazioni ${ }^{76}$. In ogni caso il «nuovo Stato anti-parlamentare» aveva come fine dichiarato di lungo termine la «riorganizzazione della vita

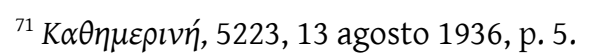

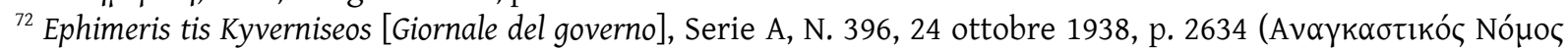

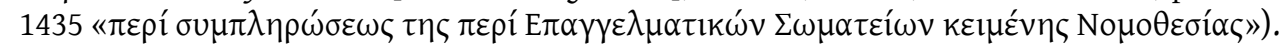

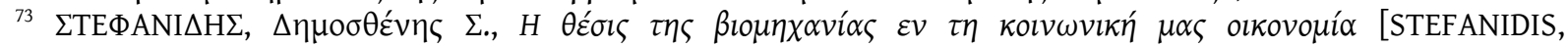

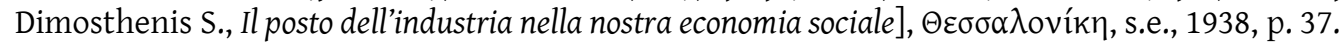

${ }^{74}$ VATIKIOTIS, Panayiotis J., op. cit., p. 161.

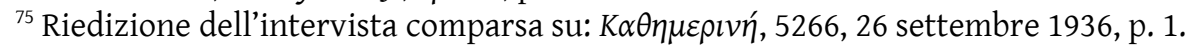

${ }^{76}$ MAZOWER, Mark, op. cit., pp. 290-291; SARANDIS, Constantine, The ideology and character of the Metaxas regime, in HIGHAM, Robin, VEREMIS, Thanos (eds.), The Metaxas Dictatorship: Aspects of Greece 1936-1940, Athens, Hellenic Foundation for Defence and Foreign Policy, 1993, pp. 156-157.
} 
sociale e politica sulla base della dottrina della cooperazione e solidarietà delle classi» ${ }^{77}$. Come dichiararono nel 1938 i giuristi teorici della "Rivoluzione del 4 Agosto", Nikolaos Koumaros e Georgios Mantzoufas, «ci sarà una rappresentanza classista del popolo, cioè dei gruppi organizzati e delle organizzazioni professionali», ma «se lo Stato del 4 Agosto intende o meno evolvere in uno Stato sindacale, ciò non è stato dichiarato» ${ }^{78}$. Metaxas e i suoi collaboratori avevano aspirato all'organizzazione collettivista della società e all'organizzazione corporativista "nazionale" del lavoro (l'istituzione di un nuovo sistema di rappresentanza orizzontale, il Gran Consiglio del Lavoro Nazionale e l'Assemblea delle Professioni), ma questa visione venne applicata su base sperimentale solo nel settore dell'agricoltura (alle Cooperative Agricole e alle Case del Contadino).

Malgrado il fatto che le ambiziose dichiarazioni di Metaxas e Zavitzianos non si fossero concretizzate pienamente, alcune misure d'interesse nazionale/"auroritarie" furono adottate nel settore dell'industria. Nel 1937 il primo maggio venne dichiarato come Giorno della Festa del Lavoro; il relativo decreto emergenziale precisava che il lavoro veniva inteso «nel suo significato ideologico nazionale» ${ }^{79}$. Nel luglio del 1940 una nuova legge d'emergenza pose le Camere Professionali e Artigianali sotto «la sovrintendenza ed il controllo» del Sottosegretariato al Lavoro, e li convertì in persone giuridiche di Diritto pubblico come «obbligatorie Unioni di professionisti di una data categoria» (il concetto di "categoria" è da interpretare in ottica corporativista). Mentre la legge istitutiva delle camere Professionali e Artigianali precisava che i membri dei loro Consigli amministrativi sarebbero stati «revocabili», la nuova legge d'emergenza stabiliva che da allora in poi un terzo dei loro membri sarebbe stato designato dal competente Sottosegretariato al Lavoro ${ }^{80}$. Le Camere commerciali e industriali non vennero modificate e nella Grecia di Metaxas non venne creato niente di paragonabile ai Reichsstände für Handel und Industrie nazionalsocialisti (Sindacati Nazionali per il Commercio e l'Industria ${ }^{81}$ ) o all'italiana Carta del Lavoro.

Nel settore dell'agricoltura, il governo di Metaxas si mosse, nell'ambito della politica dell'agricoltura pianificata, nella direzione del miglioramento della posizione materiale dei contadini, attraverso la riduzione del processo di urbanizzazione e l'istituzione di una classe agraria

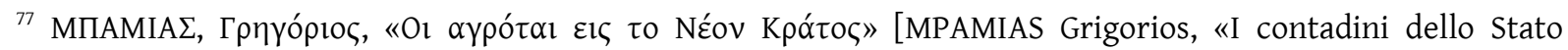

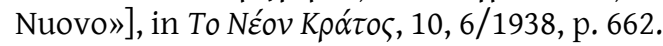

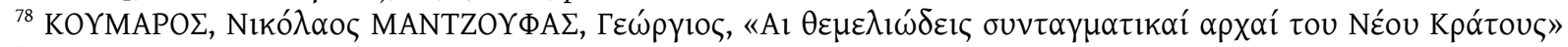
[KOUMAROS, Nicolaos D., MANTZOUFAS, Georgios A., «I principi costituzionali fondamentali dello Stato Nuovo»], in To NÉov Kpátos, 11, 7/1938, p. 818.

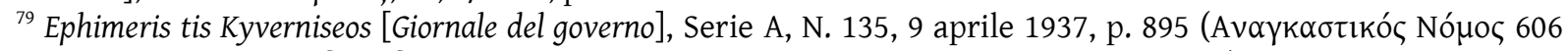

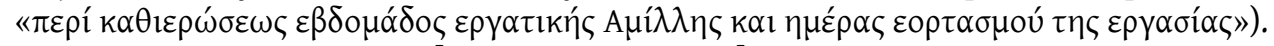

${ }_{80}$ Ephimeris tis Kyverniseos [Giornale del governo], Serie A, N. 206, 3 luglio 1940, p. 1707 (A.N. «

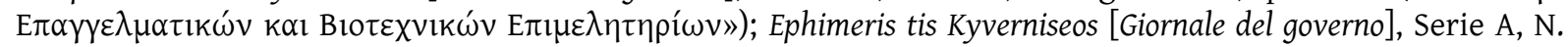

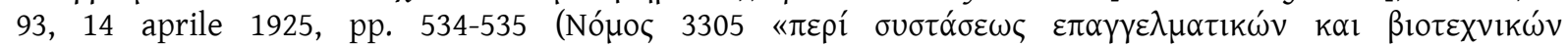
$\varepsilon \pi \imath \mu \varepsilon \lambda \eta \tau \eta \rho i ́(\omega v »)$.

${ }^{81}$ BARKAI, Avraham, Nazi Economics: Ideology, Theory, and Policy, New Haven-London, Yale University Press, 1990, p. 129.
} 
conservatrice, la quale sarebbe stata fedele agli ideali nazionali e sostenitrice del regime ${ }^{82}$. Nel marzo del 1938 venne fondata (al posto della Confederazione Panellenica delle Cooperative Agricole) la Confederazione Nazionale delle Cooperative Agricole della Grecia, la quale fu posta sotto la «diretta sovrintendenza del presidente del Governo» e divenne «l'Organizzazione suprema e universale di tutte le Organizzazioni Cooperative del Paese». La riorganizzazione centrale del movimento cooperativo ed il rafforzamento della posizione «dello Stato in modo che esso possa agire in modo più diretto sulle sue direzioni» trasformava letteralmente le cooperative in «Strumento dello Stato [sic!]». «L'intervento dello Stato», prendeva dichiaratamente come esempio la Germania e si giustificava (nel testo della legge) con l'«innalzamento del livello di vita della classe

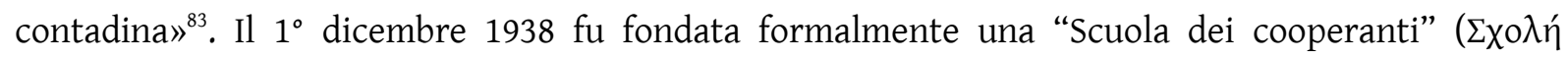

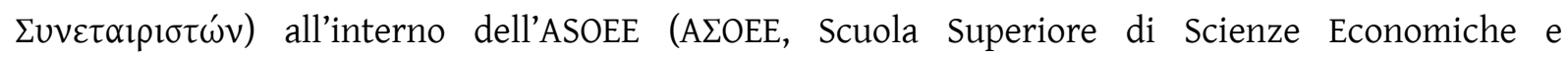
Commerciali) di Atene. Come spiegò Metaxas in un suo discorso durante l'inaugurazione della scuola, l'applicazione dell'«idea della cooperazione» (cioè del corporativismo) ebbe inizio dal mondo contadino, poiché esso costituiva lo strato sociale più basso della piramide sociale, dal quale «proviene tutto il volume della piramide fino alla cima». Metaxas espresse la propria fede nella «dottrina cooperativa»; sottolineò che «la nostra libertà è davvero molto limitata» e che «la nazione intera fatalmente costituisce un'enorme cooperativa», ma ammise che «siamo molto distanti» dall'organizzazione cooperativa «della collettività nazionale generale» ${ }^{84}$. Nell'agosto del 1939 venne istituito anche un Dipartimento delle Cooperative con a capo il segretario generale del Ministero dell'Agricoltura Babis Alivizatos. ${ }^{85}$

Ma lo strumento principale dell'esercizio della politica corporativa nel settore dell'economia agraria, erano le cosiddette Case del contadino. Nel novembre del 1938 fu promulgata l'istituzione (inizialmente affidata ai capoluoghi di ogni Regione) delle Case del contadino, che sostituirono le Camere agricole e avevano come obiettivo «l'educazione professionale e spirituale e l'illuminazione della classe agricola, la stretta collaborazione con lo Stato e l'applicazione della politica agricola tracciata di volta in volta dal Ministero dell'Agricoltura» ${ }^{86}$. Le Case del contadino avrebbero costituito - secondo Metaxas - i «nuclei combattivi» che avrebbero trasmesso alla campagna «i

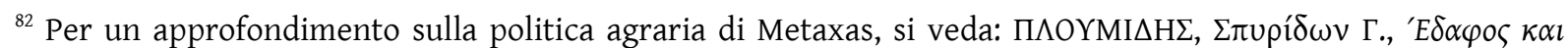

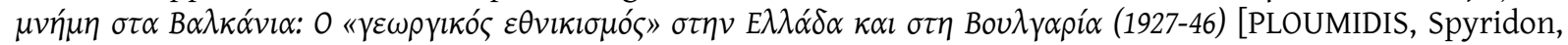

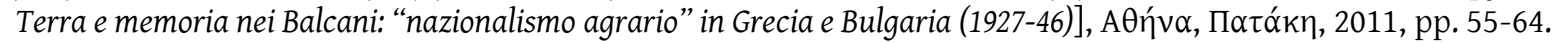

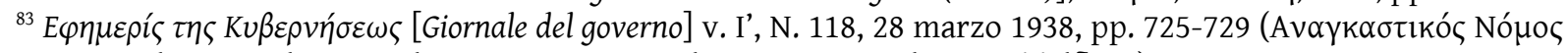

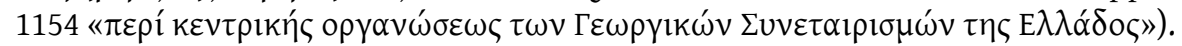

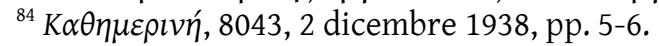

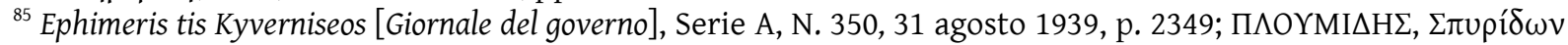

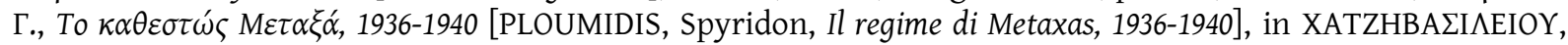

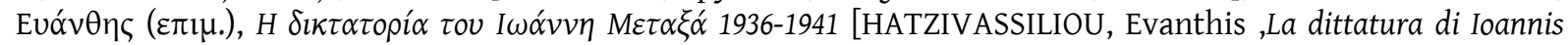

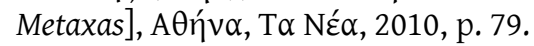

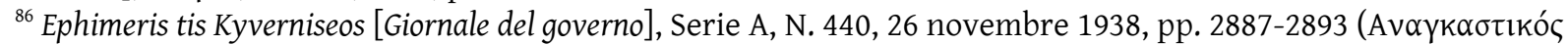

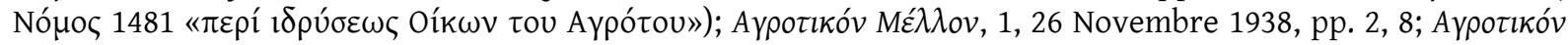

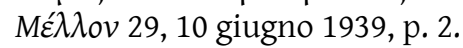


principi e lo spirito dello Stato del 4 Agosto» e sarebbero diventate «l'avanguardia militante» che avrebbe creato il «futuro agricolo migliore» del paese. ${ }^{87}$ Le Case del contadino - una creazione di Babis Alivizatos durante il ministero di Kyriakou - copiavano le (otto) corporazioni del «circolo agricolo produttivo», che vennero istituite nel 1934 nell'Italia mussoliniana dal Sottosegretario al Ministero delle corporazioni (i “sindacati”), Giuseppe Bottai nell'ambito del corporativismo (e della Carta del Lavoro del 1927) ${ }^{88}$ e le Bauernschaften (le Körperschaften nel settore della produzione, della lavorazione e del commercio di prodotti agricoli) della Germania nazionalsocialista, che erano state fondate nel settembre del $1933^{89}$. L'ispirazione per il loro nome venne probabilmente dalle casas do povo (case del popolo) portoghesi, i sindacati agricoli dell'Estado Novo di Salazar (19331974 $)^{90}$. Le Case del contadino istituzionalizzavano la classe agricola legalitaria greca e i loro membri avrebbero costituito la sua élite (l'Organizzazione politica della Classe Contadina) la quale avrebbe infuso la «solida coscienza» alle popolazioni contadine e avrebbe assunto «con vero fanatismo il lavoro della diffusione e dell'organizzazione della propaganda nelle campagne» ed il «consolidamento di una classe agraria forte dal punto di vista dell'anima e dello spirito» ${ }^{91}$. L'obiettivo di Babis Alivizatos - ricalcando letteralmente i concetti di Durrè, il quale aveva trasformato la nota fraseologia di Vilfredo Pareto e di Robert Michels adoperata dal fascismo italiano - era quello di «creare una nuova aristocrazia dei campi: l'aristocrazia delle mani "ruvide", l'aristocrazia del villaggio e della campagna». Il «Mondo Agricolo» avrebbe così acquistato la sua «"unità interna" (ed un gruppo dirigente) ed il suo posto organico dentro la cornice dello Stato del 4 Agosto» ${ }^{92}$. Malgrado le somiglianze esterne, le Case del contadino differivano in modo sostanziale

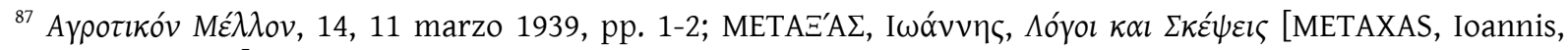
Discorsi e pensieri], op.cit., v. 2, pp. 37-45.

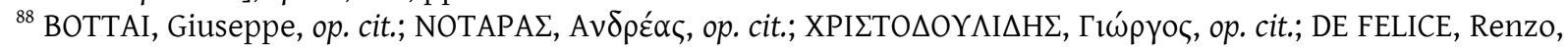
Storia dell'Italia contemporanea, vol. 3, Guerra e fascismo, 1915-1929, Napoli, ESI, 1978, pp. 406-407, 417. CANDELORO, Giorgio, Storia dell'Italia moderna, vol. IX, Il fascismo e le sue guerre, Milano, Feltrinelli, 1990, pp. 149150.

${ }^{89}$ Nella Germania nazista le (Landes-, Kreis- e specialmente le Ort-) Bauernschaften vennero fondate con legge speciale nel settembre del 1933 ed erano unioni corporativiste (Körperschaften) di produttori, elaboratori e distributori dei prodotti agricoli. Il totale di 20 Landesbauernschaften erano la «classe lavoratrice nazionale» del Terzo Reich o, come la rende in greco il Dimitrios Panou, la «Organizzazione Sindacale dell'alimentazione tedesca» (Reichsnährstand). Le Ortbauernführer si trovavano in contatto diretto con ogni contadino "associato" della loro zona. Vedi SANTORO, Cesare, Vier Jahre Hitler - Deutschland von einem Ausländergesehen,

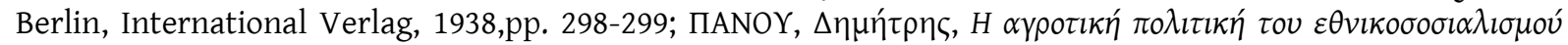
[PANOU, Dimitris, La politica agricola del nazionalsocialismo], A日ńva, s.e., 1939, pp. 9, 46, 50, 53-55.

90 I membri delle casas do povo (1933) erano sia oltivatori che proprietari terrieri. Nel 1937 la loro funzione venne assimilata con i "sindacati” industriali.

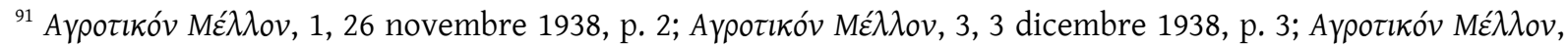

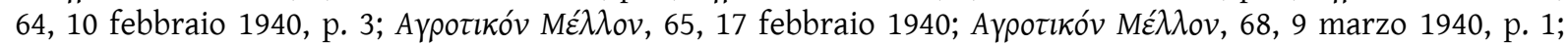

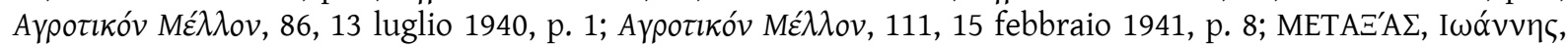

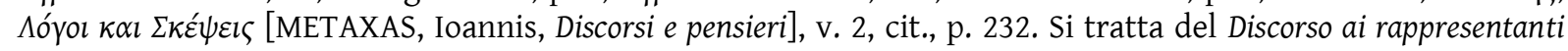
delle organizzazioni cooperative contadine del 7 gennaio 1940.

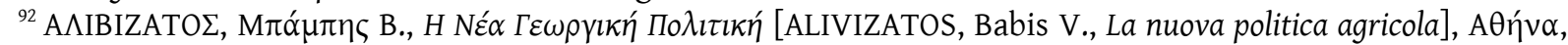

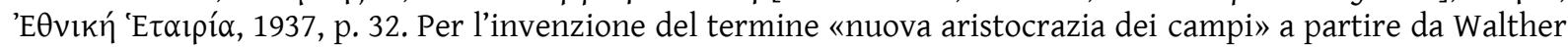
Darré vedi LANGSAM, Consuelo Walter, MITCHELL, Otis C., The World Since 1919, New York-London, Collier-
} 
dai "sindacati" agricoli nazionalsocialisti e fascisti. Così, mentre il Reichsnährstand tedesco (il "sindacato" supremo nel settore dell'alimentazione popolare) determinava il prezzo di vendita all'ingrosso e al dettaglio come anche le quote di produzione dei prodotti agricoli, aveva funzioni di controllo della qualità tanto dei prodotti agricoli freschi che di quelli lavorati e aveva la facoltà di imporre multe e pene alle industrie e ai commercianti del settore alimentare ${ }^{93}$, il legislatore greco non conferì mai competenze del genere alle Case del contadino. La politica dell'«agricoltura pianificata» di Metaxas si occupava esclusivamente della forza lavoro dell'economia e della società agricola e non arrivava fino alla regolamentazione dei termini della produzione e della distribuzione commerciale dei beni.

Fino all'entrata della Grecia nella Seconda guerra mondiale (18 ottobre 1940), il corporativismo "autoritario" aveva fatto qualche progresso, ma non aveva raggiunto lo stadio della maturità. Secondo informazioni ufficiali, fino all'agosto del 1940 furono formati alla "Scuola dei cooperanti" circa 2.000 impiegati delle organizzazioni cooperative agricole ${ }^{94}$. Durante lo stesso periodo erano in funzione Case del contadino in tutto il Peloponneso e venivano fondate gradualmente anche in tutti i capoluoghi di Regione del resto della Grecia ${ }^{95}$. La diffusione delle Case del contadino nel Peloponneso si spiega facilmente con il fatto che quella particolare zona della Vecchia Grecia era una tradizionale roccaforte degli anti-venizelisti e veniva abitata da piccoli proprietari terrieri conservatori, che erano strettamente legati alle reti di patronato dei partiti borghesi ${ }^{96}$. Al contrario l'espansione dell'istituzione della Casa del contadino in Tessaglia (la roccaforte degli agrari radicali), in Macedonia e in Tracia, dove si erano stabiliti profughi, piccoli proprietari coltivatori, fedeli al venizalismo e a una forma di governo non monarchico, era un'impresa ardua. Per quanto riguarda i contratti collettivi di lavoro, sempre secondo fonti ufficiali, la loro applicazione «fu quasi totale sul territorio e su tutte le professioni». Durante i primi tre anni del regime vennero firmati 823 contratti collettivi, dei quali 133 erano «generali, aventi cioè vigore in tutto lo Stato» e 690 «locali» ${ }^{97}$. Anche l'istituzione dell'arbitrato obbligatorio nelle differenze fra salariati e datori di lavoro sembra aver avuto un grande successo: durante il periodo 4 agosto 1938-4 agosto 1939 i servizi competenti del

Macmillan, 1971, p. 147; SOHN-RETHEL, Alfred, Economy and Class Structure of German Fascism, London, Free Association, 1987. p. 69. Per l'uso dei concetti di «élite» e «aristocrazia» di Pareto da parte Roberto Michels e dal fascismo italiano, si veda: GREGOR Anthony J., The Ideology of Fascism: The Rationale of Totalitarianism, New York-London, Free Press, 1969, pp. 217, 242; EATWELL, Roger, Fascism. A History, London, Chatto \& Windus,

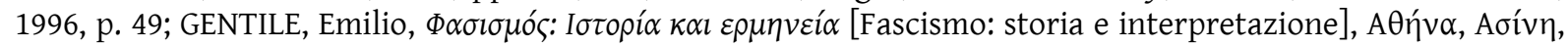
2007, pp. 26, 116.

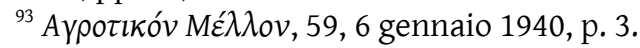

${ }^{94}$ BARKAI, Avraham, op. cit., 145-146. Va notato che, in contrasto con l'agricoltura, in cui I corpi amministrativi del Reichsnährstand organizzavano l'intero corso della trasformazione e della commercializzazione dei prodotti agricoli, industriali artigianali e le associazioni di categoria nella Germania nazista non avevano autorità sulle politiche di mercato o dei prezzi; cfr. BARKAI, Avraham, op. cit., p. 135.

${ }^{95}$ YПОҮРГЕIО ТҮПОҮ КАІ TOYРILMOY, op. cit., p. 109.

${ }^{96}$ Ibidem.

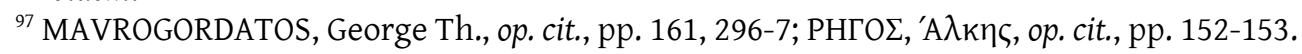


Dipartimento del Lavoro risolsero l'82\% di queste dispute (59.748 su un totale di 73.109) e soltanto il $18 \%$ dei(suddetti casi fu portato in tribunale ${ }^{98}$. Il Nuovo Stato di Metaxas poteva vantarsi di aver realizzato la cooperazione di tutte le classi «a favore dell'Economia nazionale e del benessere sociale». Il decreto emergenziale 1435/1938 definì con maggiore chiarezza che i circoli professionali per poter sottoporre allo Stato qualsiasi rivendicazione dovevano necessariamente ottenere prima «un riconoscimento speciale, in quanto "rappresentativi"». Successivamente furono riconosciuti come "rappresentativi" 1.257 circoli di operai (a paga giornaliera) e di impiegati, i quali furono di seguito subordinati a 41 Centri di operai e impiegati", e ad altrettante Segreterie inter-professionali della GSEE. In breve tempo la GSEE venne ribattezzata Confederazione Nazionale dei Lavoratori

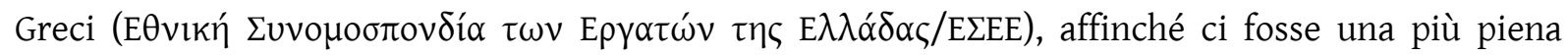
rappresentanza dei circoli professionali «all'interno dello Stato» ${ }^{99}$. L'esatta composizione e funzione di questi circoli "riconosciuti" non è nota a causa della mancanza di dati sufficienti, ma è certo che il Decreto legge 1435 mise le basi per l'istituzione dei “sindacati” corporativi di primo grado.

\section{Conclusioni}

L'applicazione del corporativismo "autoritario" nella Grecia tra le due guerre era la conseguenza del crollo del liberalismo economico - a seguito della bancarotta del 1932, a causa della crisi economica mondiale - e della acuta crisi dello Stato parlamentare liberale dopo i golpe militari consecutivi del 1933 e del 1935. Certamente quattro anni dopo l'imposizione del regime "fascisteggiante" di Ioannis Metaxas, la Grecia non era ancora uno "Stato Sindacale" e la vecchia legislazione del lavoro non era andata oltre il primo stadio del "corporativismo". Questi 1257 "rappresentativi" circoli di operai e impiegati non erano stati ancora trasformati in "sindacati" di secondo grado e non rappresentavano i datori di lavoro insieme ai loro dipendenti. Il loro numero era caotico (in confronto ad esempio ai 823 sindacati "riconosciuti" e le 22 corporazioni della vicina Italia fascista) ed era appena più piccolo del numero totale dei già esistenti libere associazioni di lavoratori autonomi e indipendenti greche (1.607), "rappresentativi" e non-riconosciuti ${ }^{100}$. Perfino a livello teorico, la propaganda delle idee e delle pratiche corporativiste "autoritarie" quasi coincise temporalmente con l'istituzione delle dittature di Kondylis e di Metaxas e si svolse parallelamente ad esse come un metodo di allineamento opportunistico e di corteggiamento del potere autoritario $^{101}$. Così come il regime di Metaxas non raggiunse mai lo stadio maturo del fascismo (o

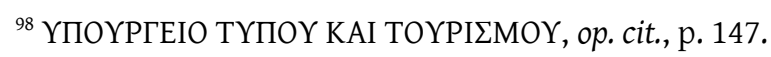

${ }^{99}$ Ibidem, p. 149.

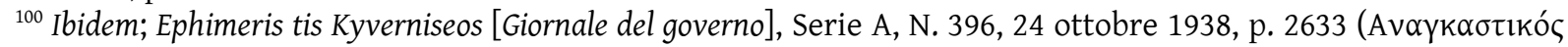
Nómoৎ 1435).

${ }^{101}$ Ad esempio le opera che erano state pubblicate da Tournakis, il promotore più inflessibile del modello corporativo italiano negli ambienti universitari, prima del 1936 riguardavano esclusivamente il commercio
} 
non fece in tempo a raggiungerlo a causa della guerra), così anche il corporativismo non divenne mai un elemento ideologico strutturale dell'economia e della politica greca. Peraltro lo stesso Metaxas diede maggiore enfasi ai valori della famiglia, della monarchia e della religione e dell'etica ortodossa, che non allo Stato. La filosofia politica di Metaxas era profondamente conservatrice e guardava più verso il passato e la tradizione piuttosto che ad una modernità alternativa, fascista o nazista ${ }^{102}$. Come è noto, il corporativismo si diffuse principalmente in paesi con una forte tradizione cattolica come l'Italia, il Portogallo, la Spagna, l'Austria e i paesi dell'America Latina.

Oltre alle ragioni soggettive, ce ne sono anche alcune oggettive che spiegano l'atrofia del corporativismo in Grecia. Innanzitutto la tarda e atrofica industrializzazione e la classe operaia fluida e disomogenea spiegano il questo esito. L'industria greca nel primo dopoguerra consisteva per la maggior parte in piccole arcaiche imprese di famiglia (nel 1930, 70.644 su un totale di 76.591 imprese, cioè una percentuale del 90\%) con capitali piccoli e un ridotto numero di dipendenti, che non superava le 6 persone ${ }^{103}$. Circa il 40\% di queste imprese erano stagionali, con un'interruzione delle attività che si protraeva dai tre ai nove mesi all'anno ${ }^{104}$. Una parte rilevante della forza lavoro era costituita dai giovani, da donne nubili (23,2\% nel 1928) e da minori (giovani “apprendisti” dai 12 ai 18 anni, il 6\% nel 1920). La maggior parte di queste lavoratrici (una percentuale del 71\% nel 1930) abbandonava l'occupazione industriale appena si sposava ${ }^{105}$. In più una parte importante degli uomini che lavorava nelle fabbriche, principalmente operai non specializzati (che ammontavano al $70 \%$ della forza lavoro del paese) considerava la sua occupazione nell'industria come provvisoria e come un trampolino di lancio per il passaggio alla piccola borghesia delle città, tramite l'apertura di un piccolo negozio o di un bar o attraverso l'assunzione nel settore pubblico ${ }^{106}$. Tutto questo impediva la formazione di una solida e durevole classe operaia che avrebbe potuto inserirsi nei

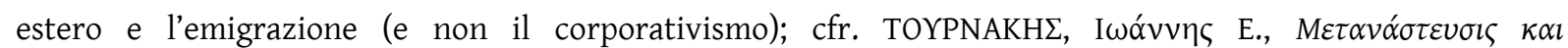

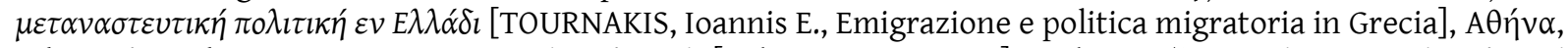

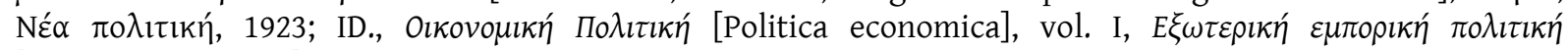

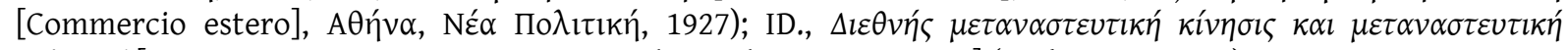

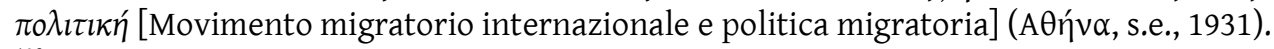

${ }_{102}$ MOSSE, George, Masses and Man: Nationalist and Fascist Perceptions of Reality, New York, Wayne State University Press, 1980, p. 187; MORGAN, Philip, Fascism in Europe, 1919-1945, London-New York: Routledge, 2003, p. 192; GRIFFIN, Roger, Modernism and Fascism: The Sense of a Beginning under Mussolini and Hitler, HoundmillsBasingstoke-New York, Palgrave Macmillan, 2007, pp. 31, 362.

${ }^{103} \Delta$ PIT $\Sigma$ A, M $\alpha \rho \gamma \alpha \rho i ́ \tau \alpha$, op. cit., p. 113; MAZOWER, Mark, op. cit., pp. 93, 95.

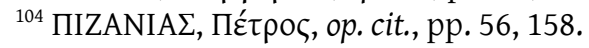

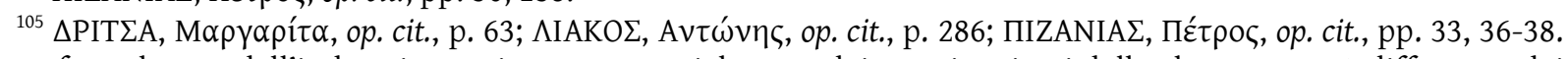
La forza lavoro dell'industria consisteva sostanzialmente dei nuovi arrivati dalle champagne. A differenza dei rifugiati provenienti dall'Asia minore, questi migranti interni, che provenivano da famiglie di piccoli proprietari terrieri, non erano indigenti e perciò non destinati a rimanere nell'industria per la maggior parte della loro vita: mantennero le loro piccole proprietà nei villaggi e con molta maggior facilità si inserivano

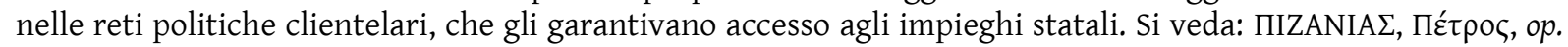
cit., p. 42.

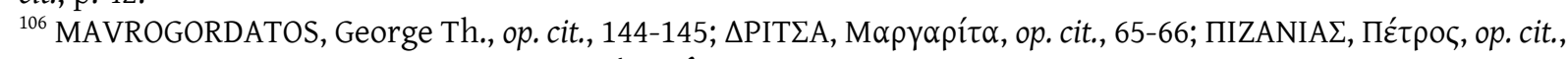

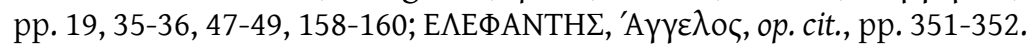


"sindacati". In definitiva la Grecia tra le due guerre può essere caratterizzata solo come esempio periferico della norma europea del corporativismo. 


\section{L'AUTORE}

Spyridon PLOUMIDIS è professore di Storia greca contemporanea presso la facoltà di Storia e Archeologia dell'Università

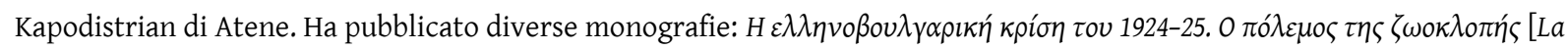

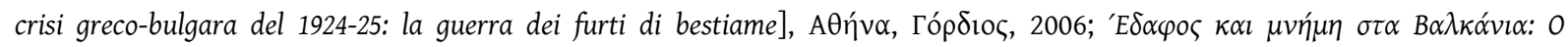

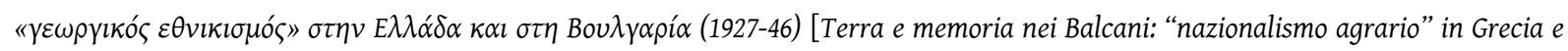

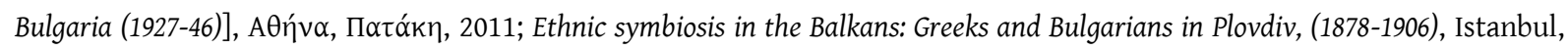

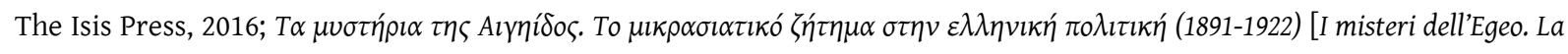

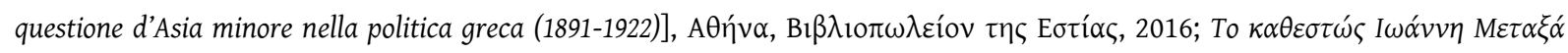

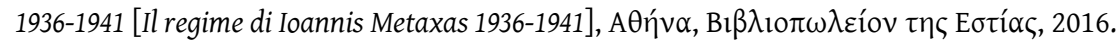

URL: < http://www.studistorici.com/progett/autori/\#Ploumidis > 